\title{
BOUNDARY CROSSING PROBABILITIES FOR STATIONARY GAUSSIAN PROCESSES AND BROWNIAN MOTION ${ }^{1}$
}

BY

\author{
JACK CUZICK
}

\begin{abstract}
Let $X(t)$ be a stationary Gaussian process, $f(t)$ a continuous function, and $T$ a finite or infinite interval. This paper develops asymptotic estimates for $P(X(t)>f(t)$, some $t \in T)$ when this probability is small. After transformation to an Ornstein Uhlenbeck process the results are also applicable to Brownian motion. In that special case, if $W(t)$ is Brownian motion, $f$ is continuously differentiable, and $T=[0, T]$ our estimate for $P(W(t)>f(t)$, some $t \in T)$ is

$$
\Lambda=\int_{0}^{T}(2 t)^{-1}\left(f(t) / t^{1 / 2}\right) \phi\left(f(t) / t^{1 / 2}\right) d t+I_{\left\{\left(f(t) / t^{1 / 2}\right)^{\prime} \mid<-T<0\right\}} \Phi^{*}\left(f(T) / T^{1 / 2}\right)
$$

provided $\Lambda$ is small. Here $\phi$ is the standard normal density and $\Phi^{*}$ is its upper tail distribution. Our approach is to find an approximate first passage density and then compute crossing probabilities as a one-dimensional integral. In the case of boundaries without cusps, our results unify and extend separate results for crossings of constant levels developed by Pickands, and Qualls-Watanabe, and crossings of rapidly increasing barriers studied by Berman. Applications are also briefly explored.
\end{abstract}

1. Introduction. Let $X(t)$ be a stationary Gaussian process and $f(t)$ a continuous function defined on some interval $T$. We shall be interested in estimating $P(X(t) \geqslant$ $f(t)$, some $t \in T$ ) when this probability is small. An important application is the estimation of boundary crossing probabilities for Brownian motion. If $W(t), t>0$, is Brownian motion, then $X(t)=e^{-t} W\left(e^{2 t}\right), t \in R$ is stationary and $P(W(t) \geqslant f(t)$, some $t \in[0, T])=P\left(X(t) \geqslant e^{-t} f\left(e^{2 t}\right)\right.$, some $\left.t \in\left(-\infty, \frac{1}{2} \ln T\right]\right)$. Our results take the form of a limit theorem: as a sequence of functions $f_{n}$ tend to infinity in an appropriate way we find constants $\Lambda_{n}$ such that $\Lambda_{n}^{-1} P\left(X(t) \geqslant f_{n}(t)\right.$, some $\left.t \in T\right) \rightarrow$ 1. When $T$ is a finite interval and the boundaries are without cusps, our results are a synthesis and extension of the work of Pickands [12], and Qualls and Watanabe [10] on the one hand who assumed the $f_{n}$ to be constant, and Berman [2], [3] who considered translations of a fixed barrier, i.e. $f_{n}(t)=n+f(t)$ on $[0, T]$ with $f(t)$ increasing. In this case when $f(t)$ is strictly increasing, Berman showed that asymptotically the barrier is only crossed in a neighborhood of the origin. This is no longer true if $T$ is an infinite interval, or if the $f_{n}$ "flatten out" as $n \rightarrow \infty$.

Received by the editors April 16, 1979 and, in revised form, February 2, 1980.

AMS (MOS) subject classifications (1970). Primary 60G15, 60F10; Secondary 60G10, 60G17, 60G40, $62 \mathrm{~L} 10$.

Key words and phrases. Boundary crossing, first passage time, Gaussian process, Brownian motion, sequential analysis, maxima.

${ }^{1}$ This research supported by a Research Fellowship awarded by the International Agency for Research on Cancer. 
Furthermore, common parametric families of interest for Brownian motion lead to multiplicative sequences of $\left\{f_{n}\right\}$, i.e. $f_{n}(t)=n f(t)$. These cases are handled by our main theorem given in $\S 3$. Our approach is to approximate the first passage density and integrate to obtain crossing probabilities. As the formula obtained includes contributions from all parts of the boundary, it is likely to be more accurate for moderately high boundaries than Berman's. Another advantage is that for Brownian motion and boundaries without cusps, all constants are readily calculated and our formula is a single definite integral of a simple integrand. However, Berman's methods also produce the asymptotic distribution of the time spent above the curve. Berman also considers boundaries with downward pointing cusps at the origin. It is possible to accommodate cusps in the present formulation and they lead to discontinuities in the approximate first passage distribution. This will be developed elsewhere. Recent work on curved boundaries can also be found in Kozačenko-Rudomanov [8] and Piterbarg-Prisjažnjuk [13].

$\S 3$ also discusses the application of the main theorem to various special cases. $\$ 2$ sets down the notation and our basic assumptions about the process $X(t)$ and the sequence of boundaries $\left\{f_{n}\right\}$. The burden of proof is given by a sequence of lemmas in $\$ 4$. $\$ 5$ examines in more detail the special case associated with Brownian motion and the Ornstein-Uhlenbeck process, while the next section studies differentiable processes and relates our results to the theory of crossings as found in the book of Cramér and Leadbetter [4]. Finally the last section looks briefly at some applications of the results.

2. Notation and assumptions. We shall make constant use of the following functions: the standard normal density $\phi(x)=\exp \left(-\frac{1}{2} x^{2}\right) / \sqrt{2 \pi}$, the normal distribution function $\Phi(x)=\int_{-\infty}^{x} \phi(y) d y, \Phi^{*}(x)=\Phi(-x)=\int_{x}^{\infty} \phi(y) d y$, and $\psi(x)=$ $\phi(x) / x$. The well-known relation, $\lim _{x \rightarrow \infty} \psi(x) / \Phi^{*}(x)=1$ will also be used without comment.

Definition. A measurable function $f(t)$ defined on $[0, \varepsilon]$ is regularly varying at the origin if there exists some $\varepsilon^{*}>0$ such that $f(t)$ is nonnegative on $\left[0, \varepsilon^{*}\right]$ and for all $s>0$

$$
\lim _{t \downarrow 0} f(s t) / f(t)=s^{\alpha}
$$

for some $\alpha$, known as the exponent of $f$. If $\alpha=0, f$ is said to be slowly varying.

A useful source of information about regularly varying functions is Seneta [14].

We shall make the following assumption about the process $X(t)$ :

Let $X(t)$ be a separable real valued stationary Gaussian process with mean zero and unit variance. Define $\rho(t)=$ $E X(0) X(t)$ and assume that the incremental variance $\sigma^{2}(t)$ $=E(X(t)-X(0))^{2}=2(1-\rho(t))$ is regularly varying at zero with exponent $\alpha>0$. We shall also assume $\sigma(t)$ is strictly increasing in some positive neighborhood of the origin. This last assumption may be dropped by employing the device used by Qualls-Watanabe but to simplify notation we shall not do so. 
Useful consequences of the assumption are recorded in the following

LEMMA 1. Let $\sigma^{2}(t)$ be a nonnegative function on $[0, \infty)$ which is regularly varying at zero with exponent $\alpha$. Then given any $\varepsilon>0$, there exists $a 0<t^{*}<1$ such that

$$
(C-\varepsilon) t^{\alpha+\varepsilon} \leqslant \sigma^{2}(t) \leqslant(C+\varepsilon) t^{\alpha-\varepsilon}, \quad t \leqslant t^{*},
$$

for some $C>0$ and

$$
(1-\varepsilon) s^{\alpha-\varepsilon} \leqslant \frac{\sigma^{2}(s t)}{\sigma^{2}(t)} \leqslant(1+\varepsilon) s^{\alpha+\varepsilon}, \quad s \geqslant 1, s t \leqslant t^{*} .
$$

Proof. These estimates follow immediately from the well-known [14, p. 2] representation of regularly varying functions: there exists a $\tau^{*}>0$ such that

$$
\sigma^{2}(t)=C(t) \exp \left\{-\int_{t}^{\tau^{*}} \frac{\alpha(u)}{u} d u\right\}, \quad 0<t<\tau^{*}
$$

with $0<C(t)<\infty$ on $\left[0, \tau^{*}\right]$ and $\lim _{t \downarrow 0} C(t)=C>0$ and $0<\alpha(u)<\infty$ on $\left[0, \tau^{*}\right]$ and $\lim _{t \downarrow 0} \alpha(u)=\alpha$.

The assumption that $\alpha>0$ guarantees that $X(t)$ is sample continuous. We shall also assume that

$$
\begin{aligned}
& \sigma^{2}(t) \text { is bounded away from zero for } t \text { bounded away from } \\
& \text { the origin. }
\end{aligned}
$$

This rules out periodic and almost periodic processes. For small enough $x>0$ let $\sigma^{-1}(x)=\inf \{t \geqslant 0: \sigma(t) \geqslant x\}$ and following Qualls and Watanabe define $\Delta(x)=\sigma^{-1}(1 / x)$. The following lemma shows that $\Delta(x)$ is regularly varying at infinity:

LEMMA 2. Let $h(t)$ be a continuous nonnegative function on $[0, \varepsilon], \varepsilon>0$, which is regularly varying at zero with positive exponent $\alpha$. For $x<\sup _{t} h(t)$, define $h^{-1}(x)$ $=\inf \{t \geqslant 0: f(t)=x\}$. Then $h^{-1}(x)$ is regularly varying with exponent $\alpha^{-1}$.

Proof. Cf. Seneta [14, p. 24].

Let $\left\{T^{n}\right\}$ be a sequence of intervals of the form $\left(-\infty, b_{n}\right],\left[a_{n}, b_{n}\right],\left[a_{n}, \infty\right)$ or $(-\infty, \infty)$, satisfying $T^{n} \subseteq T^{n+1}, n \geqslant 0$, and set $T=\cup_{n} T^{n}$. An important special case is when all the $T^{n} \equiv T$ are identical. Let $\left\{f_{n}(t)\right\}$ be a sequence of functions with $f_{n}$ defined for $t \in T^{n}$. For large $n$ we wish to estimate $P\left(X(t)>f_{n}(t)\right.$, some $\left.t \in T^{n}\right)$. We shall make the following assumptions about the sequence $\left\{f_{n}\right\}$ :

$$
\begin{aligned}
& f_{n}(t) \text { is continuous on } T_{n} . \\
& \inf _{t \in T^{n}} f_{n}(t) \rightarrow \infty \text { as } n \rightarrow \infty
\end{aligned}
$$

$$
\text { For all } \varepsilon>0, \int_{T^{n}} \psi\left(\varepsilon f_{n}(t)\right) d t \rightarrow 0 \text { as } n \rightarrow \infty \text {. }
$$

For notational simplicity define $\Delta=\Delta\left(f_{n}(t)\right)$ and let

$$
g_{n}(t, \tau)=\left[f_{n}(t+\tau \Delta)-f_{n}(t)\right] f_{n}(t)
$$


Assume that $g_{n}(t, \tau) \rightarrow g(t, \tau)$ uniformly in $t \in T$ and uniformly in $\tau$ on compact sets and that

$$
\sup _{t} g(t, \tau)<\infty \quad \text { for all } \tau \text {. }
$$

REMARKS. (i) When $T$ is a finite interval (C2) implies (C3)

(ii) The function $g_{n}(t, \tau)$ is not necessarily defined for all $t \in T$. If $T=[a, b]$ then we make the restriction $\tau \geqslant 0$ at $t=a$, and $\tau \leqslant 0$ at $t=b$. With this convention, $g_{n}$ is eventually defined and it is clear what we mean by $g(t, \tau)$. The uniformity is to be interpreted as follows: Given any $\varepsilon>0$ and $M>\infty$, there exists an $N$ such that for all $n \geqslant N$ and $|\tau|<M$

$$
\left|g_{n}(t, \tau)-g(t, \tau)\right|<\varepsilon \text { for all } t, t+\tau \Delta \in T^{n} .
$$

The uniformity assumption in (C4) places restrictions on the functional form of $g(t, \tau)$ :

LEMMA 3. Under the assumptions (C1), (C2) and (C4) we have $g(t, \tau)=C(t) \tau$ and $C(t)$ is continuous and bounded. If $\int^{\infty} \Delta\left(y^{1 / 2}\right) d y<\infty$ (e.g. when $\left.\alpha<1\right)$ then $g \equiv 0$.

Proof. Let $\Delta=\Delta\left(f_{n}(t)\right)$ and $\Delta^{*}=\Delta\left(f_{n}(t+\tau \Delta)\right)$. Then

$$
\begin{aligned}
g_{n}(t, \sigma) & =\left[f_{n}(t+\sigma \Delta)-f_{n}(t+\tau \Delta)\right] f_{n}(t)+\left[f_{n}(t+\tau \Delta)-f_{n}(t)\right] f_{n}(t) \\
& =g_{n}\left(t+\tau \Delta,(\sigma-\tau) \Delta / \Delta^{*}\right)\left\{f_{n}(t) / f_{n}(t+\tau \Delta)\right\}+g_{n}(t, \tau) .
\end{aligned}
$$

From (2.3) it follows that

$$
\frac{f_{n}(t+\tau \Delta)-f_{n}(t)}{f_{n}(t)}=\frac{g_{n}(t, \tau)}{f_{n}^{2}(t)} \rightarrow 0
$$

and an application of Lemma 2 then shows that $\Delta / \Delta^{*} \rightarrow 1$. Now let $n \rightarrow \infty$ and use the uniform convergence of the first term in (2.4) to get $g(t, \sigma)=g(t, \sigma-\tau)+$ $g(t, \tau)$. Thus $g(t, \tau)=C(t) \tau$. Again the uniform convergence of $g_{n}(t, \tau)$ in $t$ guarantees that $g$ is continuous in its first argument so that $C(t)$ is also continuous. Boundedness follows from (2.3). To establish the last assertion, assume on the contrary, that there exist $\varepsilon>0, \delta>0, t_{0}$, and $n_{0}$ such that $g_{n}(t, 1)>\varepsilon$ for $n>n_{0}$ and $t \in\left[t_{0}, t_{0}+\delta\right]$. (If $f_{n}$ is decreasing a similar argument holds for $g_{n}(t,-1)$.) Define recursively for $m \geqslant 0 y_{m}=f_{n}\left(t_{m}\right), t_{m+1}=t_{m}+\Delta\left(y_{m}\right)$. If $t_{m}-t_{0}<\delta$, then from (C4)

$$
y_{m} \geqslant y_{m-1}+\frac{\varepsilon}{y_{m-1}} \geqslant y_{0}+\varepsilon \sum_{j=1}^{m-1} \frac{1}{y_{j}} \geqslant y_{0}+\frac{\varepsilon(m-1)}{y_{m}} .
$$

Thus $y_{m} \geqslant y_{0}+\varepsilon(m-1) / y_{m}$ so that $y_{m} \geqslant y^{*} \geqslant \frac{1}{2} y_{0}+\sqrt{\varepsilon(m-1)}$ where $y^{*}$ is the largest solution of $y^{*}=y_{0}+\varepsilon(m-1) / y^{*}$. As

$$
\begin{aligned}
t_{m+1} & =\sum_{j=0}^{m} \Delta\left(y_{m}\right) \leqslant \Delta\left(y_{0}\right)+\int_{0}^{\infty} \Delta\left(\frac{1}{2} y_{0}+\sqrt{\varepsilon y}\right) d t \\
& =\Delta\left(y_{0}\right)+\varepsilon^{-1 / 2} \int_{y_{0} / 2}^{\infty} \Delta\left(y^{1 / 2}\right) d y,
\end{aligned}
$$

we may choose $n$ so large that $y_{0}$ is large enough to ensure that $t_{m}-t_{0}<\delta$ for all $m$. It follows that $\sup _{t \in\left[t_{0}, t_{0}+\delta\right]} f_{n}(t)=+\infty$ which is a contradiction. 
We need one final fact about $g(t, \tau)$ :

LEMMA 4. Let

$$
\begin{aligned}
Z_{n}=\left\{t:[t-\Delta, t+\Delta] \in T^{n}\right. \text { and either } \\
\left.\quad f_{n}(t) \leqslant \min \left(f_{n}(t+\Delta), f_{n}(t-\Delta)\right) \text { or } f_{n}(t) \geqslant \max \left(f_{n}(t+\Delta), f_{n}(t-\Delta)\right)\right\}
\end{aligned}
$$

and set $Z=\cap_{k=1}^{\infty} \overline{\bigcup_{n=k}^{\infty} Z_{n}}$. If $t \in Z$ then $C(t)=0$.

Proof. If $t \in Z$ then there exist $n_{k} \uparrow \infty$ and $t_{k} \rightarrow t$ such that either $g_{n_{k}}\left(t_{k}, \pm 1\right)>$ 0 or $g_{n_{k}}\left(t_{k}, \pm 1\right) \leqslant 0$. Since $g_{n_{k}}\left(t_{k}, \tau\right) \rightarrow g(t, \tau)=C(t) \tau$ we must have $C(t)=0$.

EXAMPLES. (i) If $X(t)$ is absolutely continuous then $\sigma^{2}(t)=k t^{2}(1+o(1))$. Taking $k=1$ we have $\Delta(x)=x^{-1}(1+o(1))$. If $f_{n}^{\prime}(t) \rightarrow f^{\prime}(t)$, continuous, then

$$
\begin{aligned}
\tau^{-1} g_{n}(t, \tau) & =\frac{f_{n}(t+\tau \Delta)-f_{n}(t)}{\tau \Delta}\left(f_{n}(t) \Delta\right) \\
& =f_{n}^{\prime}(t+\theta \tau \Delta) f_{n}(t) \Delta, \quad 0 \leqslant \theta \leqslant 1, \\
& \rightarrow f^{\prime}(t) \quad \text { since } f_{n}(t) \Delta\left(f_{n}(t)\right) \rightarrow 1 .
\end{aligned}
$$

A special case of this occurs when $f_{n}(t)=n+f(t)$, as in Berman [2], [3] and Kozačenko and Rudomanov [8].

(ii) If $X(t)$ is not absolutely continuous then $\sigma(t) / t \rightarrow \infty$ as $t \downarrow 0$ so that $x \Delta(x) \rightarrow 0$ as $x \uparrow \infty$. If $f_{n}^{\prime}(t) \rightarrow f^{\prime}(t)$ continuous, then $g(t, \tau) \equiv 0$, since

$$
\tau^{-1} g_{n}(t, \tau)=f_{n}^{\prime}(t+\theta \tau \Delta)\left[f_{n}(t) \Delta\right] \rightarrow 0 \text { as } n \rightarrow \infty .
$$

(iii) Assume $\sigma^{2}(t)=k|t|(1+o(1)), f_{n}^{\prime}(t)$ continuous, and $f_{n}^{\prime}(t) / f_{n}(t) \rightarrow C^{*}(t)$ and $C^{*}(t)$ is continuous. Taking $k=1$ gives $\Delta(x)=x^{-2}(1+o(1))$, and proceeding as before we find

$$
\begin{aligned}
\tau^{-1} g(t, \tau) & =f_{n}(t) f_{n}^{\prime}(t+\theta \tau \Delta) \Delta\left(f_{n}(t)\right), \quad 0 \leqslant \theta \leqslant 1, \\
& \rightarrow C^{*}(t)=C(t) .
\end{aligned}
$$

This case occurs if $\left\{f_{n}\right\}$ is a multiplicative sequence, i.e. $f_{n}(t)=n f(t)$.

(iv) When $\sigma^{2}(t)=t^{\alpha}(1+o(1)), \alpha>1$ so that $\Delta(x)=x^{-2 / \alpha}(1+o(1))$, and under the additional assumption that $f_{n}^{\prime}$ exists and $\lim _{n \rightarrow \infty} f_{n}^{\prime}(t+\theta \Delta) / f_{n}^{\prime}(t)=1$ uniformly in $0 \leqslant \theta \leqslant 1$ and $t$, it follows from (C4) that $\left(f_{n}(t)\right)^{1-2 / \alpha} f_{n}^{\prime}(t) \rightarrow g(t, 1)$. On integrating we obtain that

$$
f_{n}(t)=\left[f_{n}(0)^{2-2 / \alpha}+(2-2 / \alpha) \int_{0}^{t}\left(g(s, 1)+\varepsilon_{n}(s)\right) d s\right]^{1 / 2\left(1-\alpha^{-1}\right)}
$$

where $\sup _{s \in T}\left|\varepsilon_{n}(s)\right| \rightarrow_{n \rightarrow \infty} 0$. This is of the form $\left[A_{n}+f(t)+\varepsilon_{n}^{*}(t)\right]^{1 / 2\left(1-\alpha^{-1}\right)}$ where $f(t)$ and $\varepsilon_{n}^{*}(t)$ are continuously differentiable, $\varepsilon_{n}^{*}(0)=0$, and $\sup \left|\left(\varepsilon_{n}^{*}\right)^{\prime}\right| \rightarrow 0$ as $n \rightarrow \infty$. A simple example of this is the family $f_{n}(t)=n+(n \Delta(n))^{-1} f(t)$. While these examples give an idea of the general shape of the boundary, it should be noted that there is considerable variability associated with the $\varepsilon_{n}(t)$ term even when $g \equiv 0$.

3. The main theorem. Let $X_{\alpha}(t), t \geqslant 0$, be a separable Gaussian process with $X_{\alpha}(0)=0, E X_{\alpha}(t)=-\frac{1}{2} t^{\alpha}$ and $\operatorname{Var}\left(X_{\alpha}(t)-X_{\alpha}(s)\right)=|t-s|^{\alpha}$. For any measurable function $g(t, \tau)$ define 


$$
\begin{aligned}
H_{\alpha}\left(g_{t}, \theta\right) & =\frac{\int_{0}^{\infty} P\left(X_{\alpha}(\tau) \geqslant s+|g(t, \tau)|, \text { some } \tau \leqslant \theta\right) e^{s} d s}{\int_{0}^{\theta} \exp (-|g(t, \tau)|) d \tau} \\
& =E\left[\exp \left\{\sup _{\tau<\theta}\left(X_{\alpha}-|g|\right)^{+}\right\}-1\right] / \int_{0}^{\theta} \exp (-|g(t, \tau)|) d \tau
\end{aligned}
$$

and let

$$
H_{\alpha}\left(g_{t}\right)=\underset{\theta \uparrow \infty}{\lim \sup } H_{\alpha}\left(g_{t}, \theta\right)
$$

Also define

$$
\begin{gathered}
\lambda_{n}(t)=H_{\alpha}\left(g_{t}\right) \psi\left(f_{n}(t)\right) / \Delta\left(f_{n}(t)\right), \\
\lambda_{a}^{-}= \begin{cases}\Phi^{*}\left(f_{n}(a)\right) & \text { if } a>-\infty \text { and } g(a, 1)>0, \\
0 & \text { otherwise, }\end{cases}
\end{gathered}
$$

and

$$
\lambda_{b}^{+}= \begin{cases}\Phi^{*}\left(f_{n}(b)\right) & \text { if } b<\infty \text { and } g(b,-1)>0 \\ 0 & \text { otherwise. }\end{cases}
$$

Our main theorem can be stated thusly,

Theorem 1. Assume $X(t)$ satisfies $(\mathrm{P} 1)$ and $(\mathrm{P} 2)$ and $\left\{f_{n}\right\}$ satisfies $(\mathrm{C} 1)-(\mathrm{C} 4)$. Then $H_{\alpha}\left(g_{t}\right)$ is continuous in $t$ and bounded away from zero and infinity. If $T^{n}=\left[a_{n}, b_{n}\right]$ then

$$
\lim _{n \rightarrow \infty} \Lambda_{n}^{-1} P\left(X(t) \geqslant f_{n}(t), \text { some } t \in T^{n}\right)=1
$$

where

$$
\Lambda_{n}=\int_{T^{n}} \lambda_{n}(t) d t+\lambda_{a_{n}}^{-}+\lambda_{b_{n}}^{+}
$$

REMARKS. (i) When the $f_{n}$ are constant, $g(t, \tau) \equiv 0$. In that case our $H_{\alpha}(0)$ agrees with the $H_{\alpha}$ of Qualls and Watanabe [10] and our result (3.4) is their Theorem 2.1. This result was obtained earlier by Pickands [12] under further restrictions on the incremental variance $\sigma^{2}(t)$. Note that Pickands' definition of $X_{\alpha}(t)$ is slightly different from that of Qualls-Watanabe and his value for $H_{\alpha}$ also differs. However, his $\Delta(x)$ compensates for this giving the same value for $\lambda_{n}(t)$ in (3.3).

(ii) When $f_{n}(t)=n+f(t)$ and $T^{n} \equiv T$ is a finite interval, we are in a situation studied by Berman [2], [3] whose results were a by-product of the more difficult study of the time spent above a high moving barrier and are derived by a completely different method. The practical application of these results is also hampered by the difficulty in evaluating his constant $F^{\prime}(0)[2$, p. 380] and [3, p. 1024]. Our only constant is $H_{\alpha}\left(g_{t}\right)$ which can be evaluated explicitly in the important special case in which $g_{t}$ is linear and $\alpha=1$ or 2 . For $\alpha \neq 1$ or 2 it is interesting to note that the single constant $H_{\alpha}$ applies to all sets of boundaries with $g \equiv 0$ (which always occurs when $\alpha<1$ ). 
4. Proof of Theorem 1. For notational simplicity we shall take $T^{n} \equiv T=[0, \infty)$ in all arguments. Other cases are easily verified with minor modifications. Define

$$
\begin{aligned}
t_{0} & =0, \\
t_{i} & =t_{i-1}+N a \Delta\left(f_{n}\left(t_{i-1}\right)\right), \quad i=1,2,3, \ldots, \\
t_{i j} & =t_{i}+j a \Delta\left(f_{n}\left(t_{i}\right)\right), \quad j=0, \ldots, N, \\
T_{i} & =\left[t_{i}, t_{i+1}\right] .
\end{aligned}
$$

Note that $t_{i} \uparrow \infty$. In the future we shall usually suppress the argument of $\Delta$. Reference to the subscript $n$ will of ten be suppressed. Let

$$
\begin{aligned}
& x_{i}=f_{n}\left(t_{i}\right), \Delta_{i}=\Delta\left(x_{i}\right), \\
& x_{i j}=f_{n}\left(t_{i j}\right), \Delta_{i j}=\Delta\left(x_{i j}\right), \\
& \underline{\mathrm{t}}_{i}=t_{i} \text { or } t_{i+1} \text { according as } x_{i} \leqslant x_{i+1} \text { or } x_{i}>x_{i+1}, \\
& \underline{\mathrm{x}}_{i}=f_{n}\left(\mathrm{t}_{i}\right), \\
& A_{i j}=\left\{X\left(t_{i j}\right) \geqslant x_{i j}\right\}, \\
& A_{i}=\left(\bigcup_{j=0}^{N} A_{i j}\right) \cap\left\{X\left(\mathrm{t}_{i}\right)<\underline{\mathrm{x}}_{i}\right\}, \\
& A_{i}^{*}=\left\{X(t) \geqslant f_{n}(t), \text { some } t \in T_{i}, X\left(\underline{\mathrm{t}}_{i}\right)<\underline{\mathrm{x}}_{i}\right\}, \\
& A_{-}^{*}=A_{-}=\left\{X\left(a_{n}\right) \geqslant f_{n}\left(a_{n}\right)\right\}, \\
& A_{+}^{*}=A_{+}=\left\{X\left(b_{n}\right) \geqslant f_{n}\left(b_{n}\right)\right\}, \text { with the convention that } A_{-} \text {or } A_{+} \text {is empty if } \\
& a_{n}=-\infty \text { or } b_{n}=+\infty \text { resp. }
\end{aligned}
$$

We shall also use the following notation, which differs slightly from [12], [14]:

$$
\begin{aligned}
H_{\alpha}^{a}(\theta) & =\theta^{-1} \int_{0}^{\infty} P\left(X_{\alpha}(\tau) \geqslant s, \text { some } \tau \in[0, \theta] \cap I_{a}\right) e^{s} d s, \\
H_{\alpha}^{a} & =\lim _{\theta \rightarrow \infty} H_{\alpha}^{a}(\theta)
\end{aligned}
$$

where $I_{a}=\{k a\}_{k=0}^{\infty}$.

As the proof of Theorem 1 is rather long, before embarking on it, an outline of the main development is presented. We first establish that $H_{\alpha}\left(g_{t}\right)$ is continuous in $t$ (Lemma 10(iii)) and that

$$
P\left(A_{i}^{*}\right) \sim \int_{T_{i}} \frac{\psi\left(f_{n}(t)\right)}{\Delta\left(f_{n}(t)\right)} H_{\alpha}\left(g_{t}\right) d t
$$

(4.55). It is then enough to show that if $\theta \uparrow \infty$

$$
\begin{aligned}
P\left(X(t) \geqslant f_{n}(t), \text { some } t \in T^{n}\right) & \cong P\left(\bigcup_{i} A_{i}^{*} \cup A_{-}^{*} \cup A_{+}^{*}\right) \\
& \sim \sum P\left(A_{i}^{*}\right)+\lambda_{a_{n}}^{-}+\lambda_{b_{n}^{+}}^{+} .
\end{aligned}
$$

A simple way to do this is to check that $\sum_{i \neq j} P\left(A_{i}^{*} \cap A_{j}^{*}\right) / \Sigma_{i} P\left(A_{i}^{*}\right) \rightarrow 0$. Unfortunately we are unable to do this directly; the trouble arises in attempting to find adequate bounds for $P\left(\max _{t \in T_{i}} X(t) \geqslant u, \max _{t \in T_{j}} X(t) \geqslant v\right)$. Thus we are forced to consider discrete time. The proof proceeds by showing $\sum_{i \neq j} P\left(A_{i} \cap A_{j}\right) / \Sigma P\left(A_{i}\right) \rightarrow$ 0 for any $a>0$ (Lemma 8) and that $H_{\alpha}^{a}\left(g_{t}\right) \rightarrow_{a \downarrow 0} H_{\alpha}\left(g_{t}\right)$ (Lemma 10 (iv)) so that $P\left(A_{i}\right) \sim P\left(A_{i}^{*}\right)$ as $a \downarrow 0$. Care must be taken in choosing the rate at which $\theta \uparrow \infty$ as $n \uparrow \infty$. The proof develops through a series of lemmas. The first is a well-known result of Fernique [6]: 
LemMA (FerniQue). If $Y(t)$ is a mean zero Gaussian process, and $M=$ $\sup _{t \in I} E Y^{2}(t)<\infty$ for $I$ a finite interval, and $E(Y(t)-Y(s))^{2} \leqslant \psi^{2}(t-s)$ where $\psi(t)$ is continuous and nondecreasing and $\int_{1}^{\infty} \psi\left(e^{-x^{2}}\right) d x<\infty$, then there exist finite positive constants $C_{1}, C_{2}$ depending only on $M,|I|$ and $\psi$ such that

$$
P\left(\max _{t \in I} Y \geqslant u\right) \leqslant C_{1} \Phi^{*}\left(u / C_{2}\right) \text {. }
$$

The following lemma generalizes Pickands' estimate [12, Lemma 2.3]:

LEMMA 5. Let $X, Y$ be mean zero unit variance Gaussian variables with covariance $\rho$. If $a \geqslant b>0$, then

$$
P(X \geqslant a, Y \geqslant b) \leqslant 3 \psi(b) \Phi^{*}\left(a[(1-\rho) /(1+|\rho|)]^{1 / 2}\right) .
$$

Proof. We need only consider the case $|\rho|<1$. When $\rho \leqslant 0$ the result follows trivially from Slepian's lemma [16]. Assume $\rho>0$. Then

$$
\begin{aligned}
P(X \geqslant a, Y \geqslant b) & =P(X \geqslant a, Y \geqslant a)+P(X \geqslant a, b<Y<a) \\
& =P(X \geqslant a, Y \geqslant a)+\int_{b}^{a} P(X \geqslant a \mid Y=u) \phi(u) d u .
\end{aligned}
$$

However, $P(X \geqslant a \mid Y=u)=\Phi^{*}\left((a-\rho u) /\left(1-\rho^{2}\right)^{1 / 2}\right)$ is increasing in $u$ for $\rho>$ 0 . Thus (4.1) is less than

$$
\begin{aligned}
P(X \geqslant a, Y & \geqslant a)+P(X \geqslant a \mid Y=a) P(Y \geqslant b) \\
& \leqslant(1+\rho) \psi(a) \Phi^{*}\left(a\left(\frac{1-\rho}{1+\rho}\right)^{1 / 2}\right)+\Phi^{*}\left(a\left(\frac{1-\rho}{1+\rho}\right)^{1 / 2}\right) \Phi^{*}(b)
\end{aligned}
$$

by Pickands' result. Finally this is

$$
\leqslant(2+\rho) \psi(b) \Phi^{*}\left(a\left(\frac{1-\rho}{1+\rho}\right)^{1 / 2}\right)
$$

since $\psi(b) \geqslant \Phi^{*}(b)$.

LEMMA 6. Assume $a_{n}$ is nondecreasing and $b_{n}$ is nonincreasing, $n=1, \ldots, N$. Then

$$
\sum^{N} a_{n} b_{n} \leqslant \frac{1}{N}\left(\sum^{N} a_{n}\right)\left(\sum^{N} b_{n}\right)
$$

Proof. The lemma states that the sequences $\left\{a_{n}\right\}$ and $\left\{b_{n}\right\}$ are not positively correlated. As the correlation coefficient is unchanged by translations of the $a_{n}$ and $b_{n}$ we may assume $\Sigma^{N-1} a_{n}=\Sigma^{N-1} b_{n}=0$. Then $a_{N} \geqslant 0, b_{N}<0$ so that $a_{N} b_{N} \leqslant 0$ and (4.2) follows immediately from an induction argument.

Lemma 7. Assume that $a \leqslant 1$ and $N a \geqslant 2$. Then, under the conditions of Theorem 1 and the notation of the beginning of this section, there exist finite constants $D, M$ depending only on $\alpha$ and $C_{\max }=\sup _{t}|g(t, 1)|$ such that if

$$
\sup _{|\tau|<N a ; t}\left|g_{n}-g\right|<M
$$

then $\Sigma_{j} P\left(A_{i j}\right) / P\left(A_{i}\right) \leqslant D a^{-1}$ for all $n$ sufficiently large. 
Proof. Qualls and Watanabe [10, p. 586] showed that $H_{\alpha}^{a} \uparrow H_{\alpha}$ as $a \downarrow 0$. Thus $H_{\alpha}^{a}>H_{\alpha}^{1}$ for $a \leqslant 1$. When $f_{n}$ is flat we have

$$
P\left(A_{i}\right) \geqslant \frac{1}{n} P\left(\bigcup_{j<n} A_{j}\right) \geqslant \theta H_{\alpha}^{a} \Phi^{*}\left(x_{i}\right) \geqslant \theta H_{\alpha}^{1} \Phi^{*}\left(x_{i}\right)
$$

where $N a=\theta$. First assume $x_{i+1}>x_{i}$ and on $T_{i}=\left[t_{i}, t_{i+1}\right]$ define

$$
\hat{f}_{n}(t)=x_{i}+C\left(t_{i}\right) \tau x_{i}^{-1} \text { and } f_{n}^{*}(t)=x_{i}+C^{*} \tau x_{i}^{-1}
$$

where $t=t_{i}+\tau \Delta\left(x_{i}\right)$ and $C^{*}=x_{i} \theta^{-1}\left(x_{i+1}-x_{i}\right)$ so $f_{n}^{*}$ is the linear segment between $\left(t_{i}, x_{i}\right)$ and $\left(t_{i+1}, x_{i+1}\right)$. It follows from (C4), (4.3) and the identity $g(t, \tau)=$ $C(t) \tau$ that on $T_{i},\left|\hat{f}_{n}-f_{n}\right|<M x_{i}^{-1}$ and $\left|\hat{f}_{n}-f_{n}^{*}\right|<M x_{i}^{-1}$ so that

$$
\left|f_{n}-f_{n}^{*}\right|<2 M x_{i}^{-1} \equiv M^{\prime} x_{i}^{-1} \quad \text { for } t \in T_{i} \text {. }
$$

Thus

$$
\sum_{j=0}^{N} P\left(A_{i j}\right) \leqslant \sum_{j=0}^{N} \psi\left(x_{i}+\left(C^{*} j a-M^{\prime}\right) x_{i}^{-1}\right)
$$

and choosing $M^{\prime}<\frac{1}{2}$ and $n$ so large that

$$
x_{i} \geqslant \inf _{t \in T^{n}} f_{n} \geqslant 1
$$

we see that (4.6) is less than

$$
2 \psi\left(x_{i}\right) e^{M^{\prime}}\left[1+\int_{0}^{N} \exp \left(-C^{*} t a\right) d t\right] \leqslant K_{1} \psi\left(x_{i}\right)\left[1+a^{-1} \min \left(\theta, \frac{1}{C^{*}}\right)\right]
$$

where $K_{1}$ depends only on $M$.

For any $\bar{C}>0$, if $C^{*} \geqslant \bar{C}$, then (4.8) is less than $K_{1} \psi\left(x_{i}\right)\left[1+(a \bar{C})^{-1}\right]$ so that we need only show $P\left(A_{i}\right) \geqslant K_{2} \psi\left(x_{i}\right)$ in this case. For $j \leqslant N$

$$
\begin{aligned}
P\left(A_{i}\right) & \geqslant P\left(X\left(t_{i}\right)<x_{i}, X\left(t_{i}+j a \Delta\right) \geqslant x_{i}+\left(M+C_{\max } j a\right) x_{i}^{-1}\right) \\
& \geqslant \int_{x_{i}-x_{i}^{-1}}^{x_{i}} P\left(X\left(t_{i}+j a \Delta\right) \geqslant x_{i}+\left(M+C_{\max } j a\right) x_{i}^{-1} \mid X\left(t_{i}\right)=u\right) \phi(u) d u \\
& \geqslant \psi\left(x_{i}\right) P\left(X\left(t_{i}+j a \Delta\right) \geqslant x_{i}+\left(M+C_{\max } j a\right) x_{i}^{-1} \mid X\left(t_{i}\right)=x_{i}-x_{i}^{-1}\right) .
\end{aligned}
$$

The second factor of (4.9) equals

$$
\Phi^{*}\left(\frac{x_{i}+\left(M+C_{\max } j a\right) x_{i}^{-1}-\rho\left(x_{i}-x_{i}^{-1}\right)}{\left(1-\rho^{2}\right)^{1 / 2}}\right)
$$

where $\rho=\rho(j a \Delta)$. For $n$ sufficiently large and $1 \leqslant j a \leqslant 2, \rho \geqslant 0$ so that (4.10) is greater than

$$
\Phi^{*}\left((1-\rho)^{1 / 2} x_{i}+\left\{(M+1)+C_{\max } j a\right\} /\left\{(1-\rho)^{1 / 2} x_{i}\right\}\right) .
$$

Finally by (P1), $(1-\rho)^{1 / 2} x_{i} \rightarrow(j a)^{\alpha / 2} / \sqrt{2}$ as $n \rightarrow \infty$ so that $(4.11)$ is bounded below. 


$$
\begin{aligned}
& \text { When } 0 \leqslant C^{*} \leqslant \bar{C} \leqslant 1, \\
& \qquad \begin{array}{c}
P\left(A_{i}\right) \geqslant P\left(\max _{j<N} X\left(t_{i j}\right) \geqslant x_{i}+\left(M^{\prime}+C^{*} j a\right) x_{i}^{-1}, X\left(t_{i}\right)<x_{i}\right) \\
\geqslant \sup _{l<N}\left\{P \left(\max _{j<l} X\left(t_{i j}\right) \geqslant x_{i}+\left(M^{\prime}+C^{*} l a\right) x_{i}^{-1},\right.\right. \\
\left.X\left(t_{i}\right)<x_{i}+\left(M^{\prime}+C^{*} l a\right) x_{i}^{-1}\right) \\
\left.\quad-P\left(X\left(t_{i}\right) \in\left[x_{i}, x_{i}+\left(M^{\prime}+C^{*} l a\right) x_{i}^{-1}\right]\right)\right\} \\
\geqslant \sup _{l<N}\left[\Phi^{*}\left(x_{i}+\left(M^{\prime}+C^{*} l a\right) x_{i}^{-1}\right) H_{\alpha}^{1}\right. \\
\left.\quad \cdot \operatorname{la}\left\{\Delta\left(x_{i}\right) / \Delta\left(x_{i}+\left(M^{\prime}+C^{*} l a\right) x_{i}^{-1}\right)\right\}-\psi\left(x_{i}\right)\left(M^{\prime}+C^{*} l a\right)\right]
\end{array}
\end{aligned}
$$

by (4.4). Assume $M^{\prime} \leqslant 1$ and choose $l=\max _{j<N}\left\{j: C^{*} j a \leqslant 1\right\}$. Then $\bar{M}=M^{\prime}+$ $C^{*} l a \leqslant 2$ and $C^{*} l a>\frac{1}{2}$ if $l<N$ so that (4.2) is greater than

where

$$
\psi\left(x_{i}\right)\left\{K_{2} \min \left(\theta, 1 / C^{*}\right) e^{-\left(\bar{M}+\bar{M}^{2} / 2\right)}-\bar{M}\right\}
$$

$$
K_{2}=\frac{1}{6} \frac{\Delta\left(x_{i}\right)}{\Delta\left(3 x_{i}\right)} H_{\alpha}^{1}\left(\min _{x>1} \frac{\Phi^{*}(x)}{\psi(x)}\right)
$$

Thus it follows that we may choose $\bar{C}$ and $M$ depending only on $\alpha$ such that for all sufficiently large $n$ and all $C^{*} \leqslant \bar{C}$

$$
P\left(A_{i}\right) \geqslant \frac{1}{2} e^{-4} K_{2} \psi\left(x_{i}\right) \min \left(\theta, 1 / C^{*}\right) .
$$

Combining (4.8) and (4.13) and noting that $\min \left(\theta, 1 / C^{*}\right) \geqslant 1$ we see that the lemma holds when $x_{i+1} \geqslant x_{i}$. The case $x_{i+1}<x_{i}$ is handled identically except that we expand around $x_{i+1}$.

LEMMA 8. Under the assumptions of the previous lemma and the additional assumption that

$$
N a \leqslant \inf _{t \in T_{n}} f_{n}
$$

we have that for some $\varepsilon>0, K<\infty$ and all $n$ sufficiently large

$$
\frac{\sum \sum_{i \neq j} P\left(A_{i} \cap A_{j}\right)}{\sum_{i} P\left(A_{i}\right)} \leqslant K \max \left(\left(N a^{3}\right)^{-1}, a^{-2} \int_{T_{n}} \frac{\psi\left(\varepsilon f_{n}(t)\right)}{\Delta\left(f_{n}(t)\right)} d t\right) .
$$

Proof. Since $P\left(A_{i} \cap A_{j}\right) \leqslant \Sigma_{k, l} P\left(A_{i k} \cap A_{j l}\right)$ it is enough to show that

$$
\sum_{\substack{j, k, l \\ x_{j l}>x_{i k} \\ j \neq i}} P\left(A_{i k} \cap A_{j l}\right) / P\left(A_{i}\right)
$$

satisfies (4.15) for every $i$. By Lemma 5, the numerator of (4.16) is less than a constant times

$$
\sum_{\substack{j, k, l \\ x_{j l}>x_{i k} \\ j \neq i}} \psi\left(x_{i k}\right) \Phi^{*}\left(\frac{x_{j l}}{2}\left[1-\rho\left(t_{i k}-t_{j l}\right)\right]^{1 / 2}\right)
$$


Now choose $t^{*}$ so that $(2.1)$ is satisfied. When $\left|t_{i k}-t_{j l}\right|>t^{*}$ then

$$
\left[1-\rho\left(t_{i k}-t_{j l}\right)\right]^{1 / 2} \geqslant \varepsilon>0
$$

and (4.17) is less than

$$
\left(\sum_{k} \psi\left(x_{i k}\right)\right) \sum_{j, l} \Phi^{*}\left(\frac{\varepsilon}{2} x_{j l}\right)
$$

Arguing as at (2.5) and using (4.14) we have that

$$
\sup _{t \in T_{i}}\left|f_{n}(t)-x_{i}\right| / x_{i} \rightarrow 0, \quad \sup _{t \in T_{i}}\left|\Delta\left(f_{n}(t)\right)-\Delta_{i}\right| / \Delta_{i} \rightarrow 0,
$$

as $n \rightarrow \infty$ uniformly in $i$. Thus for $t \in\left[t_{i j}, t_{i j+1}\right]$ and $n$ sufficiently large

$$
\frac{\Phi^{*}\left(\lambda x_{i j}\right)}{\Delta_{i}} \leqslant \frac{2 \Phi^{*}\left(\frac{1}{2} \lambda f_{n}(t)\right)}{\Delta\left(f_{n}(t)\right)}, \quad \lambda>0,
$$

so that

$$
\Phi^{*}\left(\frac{1}{2} \varepsilon x_{j l}\right) \leqslant K a^{-1} \int_{t_{j l}}^{t_{j l+1}} \frac{\Phi^{*}\left(\frac{1}{4} \varepsilon f_{n}(t)\right)}{\Delta\left(f_{n}(t)\right)} d t
$$

by the mean value theorem. Applying (4.20) and Lemma 7 to (4.18) we see that (4.16) is less than the last term on the right-hand side of (4.15).

When $\left|t_{i k}-t_{i l}\right| \leqslant t^{*}$ we must use the regular variation of $\sigma(t)$. First assume $j>i$. Then

$$
x_{j l} \sigma\left(t_{j l}-t_{i k}\right)=\frac{\sigma\left(t_{j l}-t_{i k}\right)}{\sigma\left(\Delta_{j l}\right)} \geqslant \frac{1}{2}\left(\frac{t_{j l}-t_{i k}}{\Delta_{j l}}\right)^{\alpha / 2} \text { by Lemma } 1 .
$$

Thus

$$
\begin{aligned}
\Phi^{*}\left(\frac{x_{j l}}{2}\left[1-\rho\left(t_{i k}-t_{j l}\right)\right]^{1 / 2}\right) & \leqslant \Phi^{*}\left(\frac{1}{4}\left(\frac{t_{j l}-t_{i k}}{\Delta_{j l}}\right)^{\alpha / 2}\right) \\
& \leqslant\left(a \Delta_{j}\right)^{-1} \int_{t_{j l-1}-t_{i k}}^{t_{j l}-t_{i k}} \Phi^{*}\left(\frac{1}{4}\left(\frac{t}{\Delta_{j l}}\right)^{\alpha / 2}\right) d t \\
& =\left(\frac{\Delta_{j l}}{\Delta_{j}}\right)\left(\frac{\Delta_{i k}}{\Delta_{j l}}\right) \cdot \int_{\left(t_{j l-1}-t_{i k}\right) / \Delta_{i k}}^{\left(t_{j l}-t_{i k}\right) / \Delta_{i k}} \Phi^{*}\left(\frac{1}{4}\left[t \frac{\Delta_{i k}}{\Delta_{j l}}\right]^{\alpha / 2}\right) d t .
\end{aligned}
$$

By (4.19), the ratio $\Delta_{j l} / \Delta_{j}$ is bounded and $\Delta_{i k} / \Delta_{j l} \geqslant 1$ since $x_{i k} \leqslant x_{j l}$. One can easily check that $x \Phi^{*}(x y) \leqslant \Phi^{*}(y), x \geqslant 1, y \geqslant 1$, so that letting $y=t / 4$ and $x=\Delta_{i k} / \Delta_{j l}$ we have for $\left(t_{j l-1}-t_{i k}\right) / \Delta_{i k} \geqslant 4$ that (4.13) is bounded by a constant times $\int_{\left(t_{j i-1}-t_{i k}\right) / t_{i k}}^{\left(t_{i k}\right)} \Phi^{*}\left(\frac{1}{4} t^{\alpha / 2}\right) d t$. 
Since $\Delta_{i k} / \Delta_{i} \rightarrow 1$, this ratio is greater than one-half for $n$ large enough. Thus when $N-k>8$

$$
\begin{aligned}
\sum_{\substack{j, l \\
j>i \\
t_{i k}-t_{j l} \mid<t^{*}}} \Phi^{*}\left(\frac{1}{2} x_{j l}\left[1-\rho\left(t_{i k}-t_{j l}\right)\right]^{1 / 2}\right) & \leqslant \frac{K}{a} \int_{\left(t_{j l-1}-t_{i k}\right) / 4 \Delta_{i k}}^{\infty} \Phi^{*}\left(t^{\alpha / 2}\right) d t \\
& \leqslant \frac{K}{a} \int_{(N-k) / 8}^{\infty} \Phi^{*}\left(t^{\alpha / 2}\right) d t \equiv \frac{K b_{N-k}}{a} .
\end{aligned}
$$

It is easily checked that (4.22) is also bounded when $N-k \leqslant 8$, so that by increasing the constant if necessary we may assume (4.23) holds for all $k$. Note that $\sum_{k=0}^{\infty} b_{k}<\infty$ and $b_{k}$ is decreasing. Assume for the moment that $f_{n}$ is monotone on $\left[t_{i}, t_{i+2}\right]$. If $f_{n}$ is nondecreasing then by Lemma 6 and the estimate (4.23), we have that (4.17) is less than a constant times

$$
\frac{1}{a} \sum_{k=0}^{N} \psi\left(x_{i k}\right) b_{N-k} \leqslant \frac{1}{N a}\left(\sum_{k=0}^{N} \psi\left(x_{i k}\right)\right)\left(\sum_{k=0}^{N} b_{k}\right)
$$

which by Lemma 7 is less than $\left(K / a^{2} N\right) P\left(A_{i}\right)\left(\sum_{k=0}^{\infty} b_{k}\right)$. Thus the estimate (4.15) holds in this case. When $f_{n}$ is decreasing, no terms appear in (4.17) with $j=i+1$, and thus (4.22) is less than $K a^{-1} b_{N}$ so that (4.17) is bounded by a constant times

$$
\frac{1}{a} \sum_{k=0}^{N} \psi\left(x_{i k}\right) b_{N} \leqslant D a^{-2} P\left(A_{i}\right) b_{N}
$$

and since $N b_{N}$ is bounded the result follows. If $f_{n}$ is not monotone on $\left[t_{i}, t_{i+2}\right]$ replace it there by the linear segment

$$
\hat{f}_{n}(t)=f_{n}\left(t_{i+1}\right)+\frac{g\left(t_{i+1},\left(t-t_{i+1}\right) / \Delta_{i+1}\right)-M}{f_{n}\left(t_{i+1}\right)} .
$$

Then by (4.3)

$$
f_{n}(t)-\frac{2 M}{f_{n}\left(t_{i+1}\right)} \leqslant \hat{f}_{n}(t) \leqslant f_{n}(t), \quad t \in\left[t_{i}, t_{i+2}\right] .
$$

An elaboration of the previous argument in a manner similar to that used in Lemma 7 allows one to obtain the same bounds (4.15) in this case also (to within a multiplicative constant). An identical argument can be used when $t_{j}<t_{i}$, completing the proof of this lemma.

Corollary. Choose $0<t_{1}<t_{1}^{*}<\ldots<t_{n}<t_{n}^{*}<\ldots$ so that

$$
\left(t_{n+1}-t_{n}^{*}\right) / t_{n+1} \geqslant \varepsilon>0
$$

and let $T_{n}=\left[t_{n}, t_{n}^{*}\right] \cap I_{a}$. Then for $0<\alpha<2$ and $g(\tau) \geqslant 0$,

$$
\underset{t_{1} \uparrow \infty}{\lim \sup } \frac{\sum_{n=1}^{N} P\left(X_{\alpha}(\tau) \geqslant s+g(\tau), \text { some } \tau \in T_{n}\right)}{P\left(X_{\alpha}(\tau) \geqslant s+g(\tau), \text { some } \tau \in\left[t_{1}, t_{N}^{*}\right] \cap I_{a}\right)}<1
$$

uniformly in $N$ and $s \geqslant 0$. 
Proof. Let $\tilde{A}_{n}=\left\{X_{\alpha}(\tau) \geqslant s+g(\tau)\right.$, some $\left.\tau \in T_{n}\right\}$. Then the denominator of (4.26) is greater than or equal to

$$
P\left(\bigcup_{n=1}^{N} \tilde{A}_{n}\right) \geqslant \sum_{n=1}^{N} P\left(\tilde{A}_{n}\right)-\sum_{m \neq n} P\left(\tilde{A}_{n} \cap \tilde{A}_{m}\right) .
$$

Now $Y_{\alpha}(t)=\left[X_{\alpha}\left(e^{t}\right)+\frac{1}{2} e^{\alpha t}\right] / e^{\alpha t / 2}$ is stationary with mean zero, unit variance and covariance

$$
\begin{aligned}
\rho_{\alpha}(t) & =\frac{1}{2}\left(1+e^{\alpha t}-\left(e^{t}-1\right)^{\alpha}\right) e^{-\alpha t / 2}, \quad t>0, \\
& =1-\frac{1}{2} t^{\alpha}(1+o(1)), \quad \alpha<2 .
\end{aligned}
$$

The result is now easily obtained by applying the argument in the lemma to (4.27) to see that for $\delta=\frac{1}{4}(1-\rho(\varepsilon))$ and some $K<\infty$

$$
\frac{\sum \sum_{m \neq n} P\left(\tilde{A}_{n} \cap \tilde{A}_{m}\right)}{\sum_{n} P\left(\tilde{A}_{n}\right)} \leqslant K a^{-1} \int_{t_{1}}^{\infty} \frac{\psi\left(\delta\left[s+g(\tau)+\frac{1}{2} \tau^{\alpha}\right] / \tau^{\alpha / 2}\right)}{\Delta\left(\left[s+g(\tau)+\frac{1}{2} \tau^{\alpha}\right] / \tau^{\alpha / 2}\right)} \frac{d \tau}{\tau} \rightarrow 0 \quad \text { as } t_{1} \uparrow \infty
$$

LeMMA 9. Let $X^{*}(\cdot)$ be $X(\cdot)$ conditioned by $X(t)=f_{n}(t)-s / f_{n}(t)$ and define

$$
\begin{aligned}
X_{\alpha}^{n}(t, \tau) & =\left[X^{*}(t+\tau \Delta)-X^{*}(t)-E\left(X^{*}(t+\tau \Delta)-X^{*}(t)\right)\right] f_{n}(t)-\frac{1}{2} \tau^{\alpha} \\
& =\left(X^{*}(t+\tau \Delta)-X^{*}(t)\right)+\frac{1}{2}\left(f_{n}^{2}(t) \sigma^{2}(\tau \Delta)-\tau^{\alpha}\right)-(1-\rho(\tau \Delta)) s .
\end{aligned}
$$

Then under the conditions of Theorem 1, for $\tau$ in any compact set $\subseteq[0, \infty)$

$$
X_{\alpha}^{n}(t, \tau)-g_{n}(t, \tau) \stackrel{D}{\rightarrow} X_{\alpha}(\tau)-g(t, \tau)
$$

uniformly in $t$, where $\mathscr{D}$ denotes weak convergence.

Proof. We have that $E\left(X_{\alpha}^{n}(t, \tau)\right)=-\frac{1}{2} \tau^{\alpha}$ and

$$
\begin{aligned}
\operatorname{Var} & \left(X_{\alpha}^{n}(t, \tau)-X_{\alpha}^{n}(t, \theta)\right)=f_{n}^{2}(t) \operatorname{Var}(X(t+\tau \Delta)-X(t+\theta \Delta) \mid X(t)) \\
& =f_{n}^{2}(t) \operatorname{Var}(X(t+\tau \Delta)-X(t+\theta \Delta))\left[1-(\rho(\tau \Delta)-\rho(\theta \Delta))^{2}\right] \\
& =f_{n}^{2}(t) \sigma^{2}((\tau-\theta) \Delta)\left[1-(\rho(\tau \Delta)-\rho(\theta \Delta))^{2}\right] \\
& =|\tau-\theta|^{\alpha}(1+o(1)) \quad \text { uniformly in } t .
\end{aligned}
$$

Thus all finite dimensional distribitions of $X_{\alpha}^{n}-g_{n}$ converge to those of $X_{\alpha}-g$ uniformly in $t$. Weak convergence is obtained by checking tightness. Similar calculations can be found in [2] and are not repeated here.

The following lemma collects the essential facts associated with the functional $H_{\alpha}(\cdot)$.

LemmA 10. Let $\mathcal{C}=\mathcal{C}[0, \infty)$ and $\mathcal{C}_{0}^{\uparrow}=\bigodot \cap\{g: g(0)=0, g$ nondecreasing $\}$.

For each $g \in \mathcal{C}, a \geqslant 0$ and $\theta>0$ define the following functional on $\mathcal{C}$ :

$$
\begin{aligned}
I_{\theta, a}^{g}(f) & =\frac{\int_{0}^{\infty} I_{\left\{f(\tau)>g(\tau)+s, \text { some } \tau \in[0, \theta] \cap I_{a}\right\}} e^{s} d s}{\int_{0}^{\theta} \exp (-g(\tau)) d t} \\
& =\left[\exp \left\{\max _{\tau \in[0, \theta] \cap I_{a}}(f(\tau)-g(\tau))^{+}\right\}-1\right] / \int_{0}^{\theta} \exp (-g(\tau)) d \tau
\end{aligned}
$$


When $a=0$, we write simply $I_{\theta}^{g}(\cdot)$. Let $F$ denote expectation with respect to the measure generated on $\mathcal{C}$ by $X_{\alpha}(\tau)$. Then

(i) $I_{\theta, a}^{g}(\cdot)$ is a continuous functional on $C$ in the topology of uniform convergence on compact sets and $F\left(I_{\theta, a}^{g}\right)=H_{\alpha}^{a}(g, \theta)$.

(ii) For $g \in \mathcal{C}_{0}^{\uparrow}, H_{\alpha}(g)=\lim _{\theta \rightarrow \infty} H_{\alpha}(g, \theta)$ exists and is nonzero and finite. Furthermore $H_{\alpha}(g)=H_{\alpha}(0)$ if $\int_{0}^{\infty} \exp (-g(\tau)) d \tau=+\infty$. A similar result holds for $H_{\alpha}^{a}(g)$.

(iii) If $g_{n} \in \mathcal{C}_{0}^{\uparrow}, g_{n} \rightarrow g$ uniformly on compact sets, and

$$
\int_{0}^{\infty} e^{-g_{n}(\tau)} d \tau \underset{n \rightarrow \infty}{\rightarrow} \int_{0}^{\infty} e^{-g(\tau)} d \tau
$$

where infinity is permitted in the sequence or limit, then $H_{\alpha}\left(g_{n}\right) \rightarrow H_{\alpha}(g)$, i.e. $H_{\alpha}(\cdot)$ is continuous on $\mathcal{C}_{0}^{\top}$ in the topology $\mathcal{T}$ generated by open sets of the form

$$
\begin{aligned}
\mathcal{O}(g, N, \varepsilon) & =\left\{f: \sup _{|\tau|<N}|g-f|<\varepsilon\right\}, \\
\mathcal{O}(g, \varepsilon) & =\left\{f:\left|\int \exp (-f)-\exp (-g)\right|<\varepsilon\right\}, \text { and } \\
\mathcal{O}(M) & =\left\{f: \int \exp (-f)>M\right\} .
\end{aligned}
$$

In particular $H_{\alpha}\left(C \tau^{\beta}\right)$ is continuous in $(C, \beta)$ for $\beta>0, C \geqslant 0$ and is bounded away from zero and infinity on the compact set $0 \leqslant C \leqslant C^{*}<\infty, 0<\beta_{*} \leqslant \beta \leqslant \beta^{*}<$ $\infty$.

(iv) Let $\mathcal{T}^{*}$ be the product topology on $\mathcal{C}_{0}^{+} \times[0, \infty)$ generated by $\mathcal{T}$ and the usual topology on $[0, \infty)$. Then $H_{\alpha}^{a}(g)$ is jointly continuous in $(g, a)$ and

$$
\lim _{\substack{a \downarrow 0 \\ \theta \uparrow \infty}} H_{\alpha}^{a}(g, \theta)=H_{\alpha}(g)
$$

Proof. Since $I_{\left\{f(\tau) \geq g(\tau)+s \text {, some } \tau \in[0, \theta] \cap I_{a}\right\}}=1$ iff $\max _{\tau \in[0, \theta] \cap I_{a}}(f(\tau)-g(\tau)) \geqslant s$, one can easily see that the two expressions (4.28) and (4.29) for $I_{\theta, a}^{g}$ are equal. Statement (i) follows immediately from (4.29). To prove (ii) first let $g(\tau)=0$. In this case Pickands [12], [10] first showed that

$$
H_{\alpha}(0, \theta)=\theta^{-1} \int_{0}^{\infty} P\left(X_{\alpha}(\tau) \geqslant s, \text { some } \tau \leqslant \theta\right) e^{s} d s \underset{\theta \rightarrow \infty}{\rightarrow} H_{\alpha}(0) \equiv H_{\alpha}
$$

and that $0<H_{\alpha}<\infty$. In general, for $\delta>0$ let $\tau_{k}=\exp (\delta k)$ and $T_{k}=\left[\tau_{k}, \tau_{k+1}\right)$ $\cap[0, \theta]$. Then

$$
\begin{aligned}
& \int_{0}^{\infty} P\left(X_{\alpha}(\tau)\right.\geqslant g(\tau)+s, \text { some } \tau<\theta) e^{s} d s \\
&= \sum_{k} \int_{0}^{\infty} P\left(X_{\alpha}(\tau) \geqslant g(\tau)+s, \text { some } \tau \in T_{k},\right. \\
&\left.X_{\alpha}(\tau)<g(\tau)+s, \text { all } \tau<\tau_{k}\right) e^{s} d s \\
& \leqslant \sum_{k} \int_{0}^{\infty} P\left(X_{\alpha}(\tau) \geqslant g\left(\tau_{k}\right)+s, \text { some } \tau \in T_{k},\right. \\
&\left.X_{\alpha}(\tau)<g\left(\tau_{k}\right)+s, \text { all } \tau<\tau_{k}\right) e^{s} d s .
\end{aligned}
$$


Now make the change of variables $s^{\prime}=s+g\left(\tau_{k}\right)$ to find this equals

$$
\begin{gathered}
\sum_{k} e^{-g\left(\tau_{k}\right)} \int_{g\left(\tau_{k}\right)}^{\infty}\left\{P\left(\max _{\tau<\tau_{k+1}} X_{\alpha}(\tau) \geqslant s\right)-P\left(\max _{\tau<\tau_{k}} X_{\alpha}(\tau) \geqslant s\right)\right\} e^{s} d s \\
\leqslant \sum_{k} e^{-g\left(\tau_{k}\right)}\left[P\left(\tau_{k+1}\right)-P\left(\tau_{k}\right)\right]
\end{gathered}
$$

where $P(t)=\int_{0}^{\infty} P\left(\max _{\tau \leqslant t} X_{\alpha}(\tau) \geqslant s\right) e^{s} d s$.

Now by (4.31) $P(t) / t$ is bounded for $t>1$ and given any $\varepsilon>0$ we may choose $\theta^{*}=$ some $\tau_{k}$ so that

$$
\left|P(t) / t-H_{\alpha}\right|<\varepsilon \quad \text { for } t>\theta^{*} .
$$

Thus (4.34) is bounded by

$$
P\left(\theta^{*}\right)+\sum_{\tau_{k}>\theta^{*}} e^{-g\left(\tau_{k}\right)} e^{k \delta}\left[H_{\alpha}\left(e^{\delta}-1\right)+2 \varepsilon e^{\delta}\right] .
$$

For any $\delta>0$ we may choose $\theta^{*}$ so that $2 \varepsilon e^{\delta}<H_{\alpha}\left(e^{\delta}-1\right)^{2}$. Then the second term of (4.36) is less than $e^{2 \delta} H_{\alpha} \int_{0}^{\theta} e^{-g(\tau)} d \tau$. From this it follows that $H_{\alpha}(g, \theta)$ is bounded and so $H_{\alpha}(g)$ is finite. When $\int_{0}^{\infty} \exp (-g(\tau)) d \tau<\infty$, it follows that $\lim _{\theta \rightarrow \infty} H_{\alpha}(g, \theta)$ exists since the numerator and denominator in (3.1) are both increasing and both the ratio and denominator are bounded. When $\int_{0}^{\infty} \exp (-g(\tau)) d \tau=\infty$, by taking $\delta$ sufficiently small we see that $H_{\alpha}(g) \leqslant H_{\alpha}(0)$. It remains to provide the same lower bound in this case. First assume $\alpha<2$, let $\tau_{k}^{*}=\exp (\delta(k+1-\xi))$ for $\xi>0$ and let $T_{k}^{*}=\left[\tau_{k}, \tau_{k}^{*}\right] \cap I_{a}, a>0$. Then

$$
\left(\tau_{k+1}-\tau_{k}^{*}\right) / \tau_{k+1}=1-\exp (-\delta \xi)>0 \text {. }
$$

By the corollary to Lemma 8 , for any $\varepsilon>0$, there exists a $\theta^{*}=$ some $\tau_{k}$ such that (4.32) is greater than

$$
\begin{aligned}
&(1-\varepsilon) \int_{0}^{\infty} \sum_{\theta^{*}<t_{k}<\theta} P\left(X_{\alpha}(\tau) \geqslant g(\tau)+s, \text { some } \tau \in T_{k}^{*}\right) e^{s} d s \\
&=(1-\varepsilon)\left\{\sum_{\theta^{*}<t_{k}<\theta} e^{-g\left(\tau_{k+1}\right)} \int_{0}^{\infty} P\left(X_{\alpha}(\tau) \geqslant s, \text { some } \tau \in T_{k}^{*}\right) e^{s} d s\right. \\
&\left.\quad-\sum_{\theta^{*}<t_{k}<\theta} e^{-g\left(\tau_{k+1}\right)} \int_{0}^{g\left(\tau_{k+1}\right)} P\left(X_{\alpha}(\tau) \geqslant s, \text { some } \tau \in T_{k}^{*}\right) e^{s} d s\right\}
\end{aligned}
$$

Since $Y_{\alpha}(t)=\left(X_{\alpha}\left(e^{t}\right)+\frac{1}{2} e^{\alpha t}\right) / e^{\alpha t / 2}$ is stationary we have that

$$
\begin{aligned}
P\left(X_{\alpha}(\tau) \geqslant s,\right. & \text { some } \left.t \in T_{k}^{*}\right) \leqslant P\left(X_{\alpha}(\tau) \geqslant 0, \text { some } \tau \in T_{k}^{*}\right) \\
& =P\left(Y_{\alpha}(\tau) \geqslant \frac{1}{2} e^{\alpha \tau / 2}, \text { some } \tau \in[k \delta,(k+1-\xi) \delta]\right) \\
& \leqslant C_{2} \Phi^{*}\left(C_{1}^{-1} e^{\alpha \delta k / 2}\right)
\end{aligned}
$$

for some finite constants $C_{1}$ and $C_{2}$ by Fernique's lemma. Substituting (4.38) into the second term of (4.37), we see that this term remains bounded as $\theta \rightarrow \infty$. Thus we need only consider the first term of (4.37). Pickands [12] has shown that $H_{\alpha}^{a}(\theta) \rightarrow_{\theta \rightarrow \infty} H_{\alpha}^{a} \rightarrow_{a \downarrow 0} H_{\alpha}$. Thus given $\varepsilon>0$, we may choose $a$ and $\theta^{*}$ such that $\left|H_{\alpha}^{a}(\theta)-H_{\alpha}\right|<\varepsilon$ for $\theta>\theta^{*}$. Thus the first term of (4.37) is greater than

$$
(1-2 \varepsilon)^{2} H_{\alpha} \sum_{\theta^{*}<\tau_{k}<\theta} e^{-g\left(\tau_{k+1}\right)}[\exp \{(k+1-\xi) \delta\}-\exp (k \delta)] .
$$


However, the summand in (4.39) is greater than

$$
e^{(1-\xi) \delta}-1 / e^{\delta}\left(e^{\delta}-1\right) \int_{\tau_{k+1}}^{\tau_{k+2}} \exp (-g(\tau)) d \tau
$$

from which the lower bound easily follows.

When $\alpha=2, X_{\alpha}(\tau)=\tau\left(X-\frac{1}{2} \tau\right)$ where $X$ is a standard normal variable. Then letting $\tau=X$ for $0 \leqslant X \leqslant \theta$

$$
\begin{aligned}
E\left(\exp \left\{\max _{\tau<\theta}\left[X_{\alpha}(\tau)-g(\tau)\right]\right\}\right) & \geqslant E\left(\exp \left\{\frac{1}{2} X^{2}-g(X)\right\} I_{0<X<\theta}\right) \\
& =\frac{1}{\sqrt{2 \pi}} \int_{0}^{\theta} e^{-g(\tau)} d \tau
\end{aligned}
$$

so that $H_{2}(g) \geqslant 1 / \sqrt{2 \pi}=H_{2}(0)$ in this case also.

The proof for $H_{\alpha}^{a}(g), a>0$, follows the same development but is easier.

Proof OF (iii). If $\int_{0}^{\infty} \exp \left(-g_{n}(t)\right) d t=\infty$ for $n \geqslant n_{0}$, then $H_{\alpha}\left(g_{n}\right)=H_{\alpha}(g)=$ $H_{\alpha}, n \geqslant n_{0}$. If $\int_{0}^{\infty} \exp (-g(t)) d t<\infty$, then we need only show that $P_{\alpha}\left(g_{n}\right) \rightarrow P_{\alpha}(g)$ where

$$
P_{\alpha}(h, \theta)=\int_{0}^{\infty} P\left(X_{\alpha}(t) \geqslant s+h(\tau), \text { some } \tau \leqslant \theta\right) e^{s} d s
$$

and

$$
P_{\alpha}(h)=\lim _{\theta \rightarrow \infty} P_{\alpha}(h, \theta)
$$

We have that for all $\theta^{*}$

$$
\begin{aligned}
\left|P_{\alpha}\left(g_{n}\right)-P_{\alpha}(g)\right| \leqslant & \left|P_{\alpha}\left(g_{n}\right)-P_{\alpha}\left(g_{n}, \theta^{*}\right)\right|+\left|P_{\alpha}\left(g_{n}, \theta^{*}\right)-P_{\alpha}\left(g, \theta^{*}\right)\right| \\
& +\left|P_{\alpha}\left(g, \theta^{*}\right)-P_{\alpha}(g)\right| .
\end{aligned}
$$

As in the estimates following (4.32) we see that for $\theta^{*}>1$, there exists $C<\infty$ such that

$$
\begin{aligned}
\mid P_{\alpha}\left(g_{n}\right)- & P_{\alpha}\left(g_{n}, \theta^{*}\right) \mid \leqslant C \int_{\theta^{*}}^{\infty} e^{-g_{n}(t)} d t \\
& =C\left\{\int_{0}^{\infty} e^{-g_{n}(t)} d t-\int_{0}^{\theta^{*}} e^{-g_{n}(t)} d t\right\} \\
& \leqslant C\left\{\left|\int_{0}^{\infty} e^{-g_{n}(t)}-e^{-g(t)} d t\right|+\int_{0}^{\theta^{*}}\left|e^{-g_{n}(t)}-e^{-g(t)}\right| d t+\int_{\theta^{*}}^{\infty} e^{-g(t)} d t\right\} .
\end{aligned}
$$

Now choose $\theta^{*}$ so that the last term in (4.40) and (4.41) is small and then use the assumptions to see that the remaining terms are small for $n \geqslant n_{0}\left(\theta^{*}\right)$.

The remaining case to consider is when $\int_{0}^{\infty} \exp \left(-g_{n}(\tau)\right) d \tau<\infty$ but tends to infinity. In this case $H_{\alpha}\left(g_{n}\right) \rightarrow H_{\alpha}=H_{\alpha}(g)$ can be checked by a minor adaptation of the argument ensuing from (4.33).

Proof of (iv). We shall first show that

$$
\lim _{\theta \rightarrow \infty} H_{\alpha}^{a}(g, \theta)=H_{\alpha}^{a}(g) \text { uniformly for }(g, a) \text { in compact sets. }
$$

Note that $H_{\alpha}^{a}(\theta) \rightarrow_{\theta \rightarrow \infty} H_{\alpha}^{a}$ uniformly for $0<a \leqslant a^{*}<\infty$ from the results of 
Qualls-Watanabe [10, Lemma 2.3]. Using this estimate in place of (4.35), (4.42) can be derived by an argument similar to that used in (ii). To show that $H_{\alpha}^{a}(g)$ is jointly continuous assume $a_{n} \rightarrow a$ and $g_{n} \rightarrow g$. Then, given any $\varepsilon>0$, by (4.42) we may choose $\theta$ so large that (insuring that $\theta / a$ is not an integer when $a>0$ )

$$
\begin{gathered}
\left|H_{\alpha}^{a}(g)-H_{\alpha}^{a}(g, \theta)\right|<\varepsilon, \\
\left|H_{\alpha}^{a_{n}}\left(g_{n}\right)-H_{\alpha}^{a_{n}}\left(g_{n}, \theta\right)\right|<\varepsilon \text { for all } n .
\end{gathered}
$$

Since $\sup _{|\tau|<\theta}\left|g_{n}(\tau)-g(\tau)\right| \rightarrow_{n \rightarrow \infty} 0$, we have that

$$
\left|H_{\alpha}^{a_{n}}\left(g_{n}, \theta\right)-H_{\alpha}^{a_{n}}(g, \theta)\right| \underset{n \rightarrow \infty}{\rightarrow} 0 \text { uniformly in } a_{n} .
$$

Thus to establish joint continuity it is enough to show $\left|H_{\alpha}^{a_{n}}(g, \theta)-H_{\alpha}^{a}(g, \theta)\right| \rightarrow 0$ which is a consequence of the dominated convergence theorem. When $a>0$ our conditions guarantee that $\left[\theta / a_{n}\right]$ is ultimately a constant so that

$$
\max _{\substack{\tau<\theta \\ \tau \in I_{a_{n}}}}\left(X_{\alpha}(t)-g(t)\right) \stackrel{\text { a.s. }}{\rightarrow} \max _{\substack{\tau<\theta \\ \tau \in I_{a}}}\left(X_{\alpha}(t)-g(t)\right) .
$$

The limit (4.30) now follows immediately from (4.42) and the continuity of $H_{\alpha}^{a}(g)$ in $a \geqslant 0$.

REMARK. It can also be shown that in fact $H_{\alpha}^{a}(g, \theta) \rightarrow_{\theta \rightarrow \infty} H_{\alpha}^{a}(g)$ uniformly in $(a, g)$ on compact sets and $\alpha \geqslant \alpha^{*}>0$ so that $H_{\alpha}^{a}(g)$ is jointly continuous in $(a, g, \alpha)$. In particular, $H_{\alpha}$ is a continuous function of $\alpha$.

Proof of Theorem 1. First assume $f_{n}$ is nondecreasing on $T^{n}$ for all large $n$. We begin with an upper bound:

$$
P\left(X(t) \geqslant f_{n}(t), \text { some } t \in T^{n}\right)=P\left(X\left(a_{n}\right) \geqslant f_{n}\left(a_{n}\right)\right)+P\left(\bigcup_{i} A_{i}^{*}\right)
$$

and

$$
P\left(\cup A_{i}^{*}\right) \leqslant \sum_{i} P\left(A_{i}^{*}\right)
$$

Choose $i_{n}$ such a way that $t_{i_{n}} \rightarrow t$, and dropping $n$ from the notation we have

$$
\begin{aligned}
& P\left(A_{i}^{*}\right)= P\left(X\left(t_{i}\right)<x_{i}, X\left(t_{i}+\tau \Delta\right) \geqslant f_{n}\left(t_{i}+\tau \Delta\right), \text { some } \tau \leqslant \theta\right) \\
&= \int_{-\infty}^{0} P\left(X\left(t_{i}+\tau \Delta\right) \geqslant f_{n}\left(t_{i}+\tau \Delta\right),\right. \\
&\text { some } \left.\tau \leqslant \theta \mid X\left(t_{i}\right)=x_{i}+s x_{i}^{-1}\right) \phi\left(x_{i}+s x_{i}^{-1}\right) x_{i}^{-1} d s \\
&=\psi\left(x_{i}\right) \int_{0}^{\infty} P\left(X_{\alpha}^{n}\left(t_{i}, \tau\right) \geqslant g_{n}\left(t_{i}, \tau\right)+\frac{1}{2}\left(x_{i}^{2} \sigma^{2}(\tau \Delta)-\tau^{\alpha}\right)\right. \\
&+\rho(\tau \Delta) s, \text { some } \tau \leqslant \theta) e^{s} \exp \left(-\frac{1}{2}\left(s x_{i}^{-1}\right)^{2}\right) d s .
\end{aligned}
$$

Now $x_{i}^{2} \sigma^{2}(\tau \Delta)-\tau^{\alpha}=\sigma^{2}(\tau \Delta) / \sigma^{2}(\Delta)-\tau^{\alpha} \rightarrow 0$ uniformly in all $t$ and $\tau$ on compact sets. Thus $g_{n}^{*}\left(t_{i}, \tau\right)=g_{n}\left(t_{i}, \tau\right)+\frac{1}{2}\left(x_{i}^{2} \sigma^{2}(\tau \Delta)-\tau^{\alpha}\right) \rightarrow g(t, \tau)$ uniformly in all $t$ and $\tau$ on compact sets. Also for fixed $\theta$, given any $\varepsilon>0$, we may choose $n$ so large that $\inf _{\tau<\theta} \rho(\tau \Delta) \geqslant 1-\varepsilon$. Thus the integral in (4.46) is bounded by 


$$
\begin{aligned}
\int_{0}^{\infty} P\left(X_{\alpha}^{n}\left(t_{i}, \tau\right) \geqslant\right. & \left.g_{n}^{*}\left(t_{i}, \tau\right)+(1-\varepsilon) s, \text { some } \tau \leqslant \theta\right) e^{s} d s \\
& =E \exp \left\{\max _{\tau<\theta}\left[X_{\alpha}^{n}\left(t_{i}, \tau\right)-g_{n}^{*}\left(t_{i}, \tau\right)\right] /(1-\varepsilon)\right\}-1 .
\end{aligned}
$$

Fernique's lemma guarantees that this is dominated and our weak convergence results, Lemmas 9 and 10(i), show that for $\theta$ fixed and $n \rightarrow \infty$ (4.47) tends to

$$
E \exp \left\{\max _{\tau<\theta}\left[X_{\alpha}(\tau)-g(t, \tau)\right] /(1-\varepsilon)\right\}-1 .
$$

As $\varepsilon>0$ was arbitrary we see that for fixed $\theta$, the lim sup of the integral in (4.46) is less than

$$
E\left\{\exp \left[\max _{\tau<\theta} X_{\alpha}(\tau)-g(t, \tau)\right]\right\}-1 .
$$

Replacing $\rho(\tau \Delta)$ by 1 and $\left(s / x_{i}\right)^{2}$ by $\left(\max _{\tau<\theta}\left(X_{n}^{\alpha}-g_{n}^{*}\right) / \gamma_{n}\right)^{2}$ where $\gamma_{n}=$ $\inf _{t \in T_{n}} f_{n}(t)$, a lower bound for the integral in (4.46) is

$$
E \exp \left\{\left[-\max _{\tau<\theta}\left(X_{\alpha}^{n}-g_{n}^{*}\right)^{2} / \gamma_{n}^{2}\right]\left[\exp \left\{\max _{\tau<\theta}\left(X_{\alpha}^{n}-g_{n}^{*}\right)\right\}-1\right]\right\}
$$

which tends to (4.48) since $\gamma_{n} \rightarrow \infty$.

It then follows by a diagonal argument that there exists $\theta_{n} \uparrow \infty$ such that if $1 \leqslant \theta \leqslant \theta_{n}$

$$
\left|\frac{P\left(A_{i}^{*}\right)}{\psi\left(x_{i}\right) \int_{0}^{\theta} e^{-g\left(t_{i}, \tau\right)} d \tau}-H_{\alpha}\left(g_{t}, \theta\right)\right| \rightarrow 0
$$

uniformly in $t$ as $n \rightarrow \infty$. Now choose $\theta \uparrow \infty$ so slowly as $n \rightarrow \infty$ that

$$
\begin{gathered}
\sup _{|\tau|<\theta ; t}\left|g_{n}(t, \tau)-g(t, \tau)\right| \rightarrow 0 \quad \text { by (C4), } \\
\sup _{t \in T_{i}} \Delta\left(f_{n}(t)\right) / \inf _{t \in T_{i}} \Delta\left(f_{n}(t)\right) \rightarrow 1 \quad \text { uniformly in } i \text { (as at (4.19)) }
\end{gathered}
$$

and

$$
\theta\left(\inf _{t \in T_{n}} f_{n}\right)^{-1} \rightarrow 0 \text { by (C2). }
$$

Then for any $u=t_{i}+\tau \Delta \in T_{i}$

$$
\begin{aligned}
\psi\left(f_{n}(u)\right) & =\psi\left(x_{i}+g_{n}\left(t_{i}, \tau\right) / x_{i}\right) \\
& =\psi\left(x_{i}\right) e^{-g_{n}\left(t_{i} \tau\right)} e^{-\left(g_{n} / x_{i}\right)^{2} / 2}\left(1+g_{n} / x_{i}^{2}\right)^{-1} \\
& \sim \psi\left(x_{i}\right) e^{-g\left(t_{i}, \tau\right)} \quad \text { by }(4.50) \text { and }(4.52)
\end{aligned}
$$

and since $t_{i+1}=t_{i}+\theta \Delta$, we find using (4.51) and (4.53) that

$$
\int_{t_{i}}^{t_{i+1}} \frac{\psi\left(f_{n}(t)\right)}{\Delta\left(f_{n}(t)\right)} d t \sim \psi\left(x_{i}\right) \int_{0}^{\theta} e^{-g\left(t_{i}, \tau\right)} d \tau
$$

uniformly in $i$. Combining (4.49) and (4.54) and choosing $\theta \uparrow \infty$ so slowly as to satisfy (4.49), (4.50), (4.51) and (4.52) yields

$$
P\left(A_{i}^{*}\right) / \int_{t_{i}}^{t_{i+1}} \frac{\psi\left(f_{n}(t)\right)}{\Delta\left(f_{n}(t)\right)} d t \rightarrow H_{\alpha}\left(g_{t}\right) \quad \text { uniformly in } t .
$$


This result also holds if $g\left(t_{i}, \tau\right)<0, \tau \geqslant 0$. If $g\left(t_{i+1}, \tau\right) \geqslant 0$ for negative $\tau$ we expand all expressions around $t_{i+1}$. If this fails to hold, replace $f_{n}(t)$ on $\left[t_{i}, t_{i+1}\right]$ by the segment joining $x_{i}$ and $x_{i+1}$, and then argue as in Lemma 7 to show this adjustment is asymptotically negligible. By Lemma 10(ii) and (iii), $H_{\alpha}\left(g_{t}\right)$ is uniformly continuous in $t$ and is never zero so that

$$
\limsup _{n \rightarrow \infty} \frac{P\left(X(t) \geqslant f_{n}(t), \text { some } t \in T^{n}, X\left(a_{n}\right)<f_{n}\left(a_{n}\right)\right)}{\int_{T^{n}} \lambda_{n}(t) d t} \leqslant 1 .
$$

To find a lower bound for (4.43) the left-hand side of (4.44) is greater than

$$
P\left(\cup A_{i}\right) \geqslant \sum_{i} P\left(A_{i}\right)-\sum_{i \neq j} P\left(A_{i} \cap A_{j}\right)
$$

for all $a>0$. Taking $\theta=N a \uparrow \infty$ and holding $a$ fixed, Lemma 8 shows that the two sides of (4.57) are asymptotically equivalent. The argument leading to (4.55) also shows that

$$
P\left(A_{i}\right) / \int_{t_{i}}^{t_{i+1}} \frac{\psi\left(f_{n}(t)\right)}{\Delta\left(f_{n}(t)\right)} d t \rightarrow H_{\alpha}^{a}\left(g_{t}\right) \quad \text { uniformly in } t
$$

so that

$$
\liminf _{n \rightarrow \infty} \frac{P\left(X(t) \geqslant f_{n}(t), \text { some } t \in T^{n}, X\left(a_{n}\right)<f_{n}\left(a_{n}\right)\right)}{\int_{T^{n}} \lambda_{n}^{a}(t) d t} \geqslant 1
$$

where $\lambda_{n}^{a}=\left(\psi\left(f_{n}(t)\right) / \Delta\left(f_{n}(t)\right)\right) H_{\alpha}^{a}\left(g_{t}\right)$.

Finally by Lemma 10 (iv), $H_{\alpha}^{a}\left(g_{t}\right)$ is continuous in $a$, uniformly over the compact set $\left(g_{t}\right)_{t \in T^{n}}$, and since it is never zero we have

$$
\lim _{a \downarrow 0} \int_{T^{n}} \lambda_{n}^{a}(t) d t / \int_{T^{n}} \lambda_{n}(t) d t=1
$$

so that $P\left(\cup A_{i}^{*}\right) \sim \int_{T^{n}} \lambda_{n}(t) d t$. If $g\left(a_{n}, 1\right)=0$ then $P\left(X\left(a_{n}\right) \geqslant f_{n}\left(a_{n}\right)\right)$ is negligible compared to $P\left(A_{0}^{*}\right)$ and may be ignored. However if $g\left(a_{n}, 1\right)>0$, this term may not be small and we must add $\lambda_{a_{n}}^{-}$. By reversing the time axis and replacing $\lambda_{a_{n}}^{-}$with $\lambda_{b_{n}}^{+}$we have also established the theorem when $f_{n}$ is nonincreasing for all large $n$. In general $f_{n}$ may have interior local maxima and minima and if $x_{i}<\min \left(x_{i-1}, x_{i+1}\right)$ then $\left\{X\left(t_{i}\right) \geqslant x_{i}\right\} \nsubseteq \cup A_{j}^{*}$. Howvever Lemma 4 shows that at such points $\lim _{n} C\left(t_{i}\right)=0$ and, as with the endpoint case, the term $P\left(X\left(t_{i}\right) \geqslant x_{i}\right)$ is asymptotically negligible. By using the remark after (4.55) the theorem is established in this case also.

5. The case $\alpha=1$ and Brownian motion. The case $\alpha=1$ is particularly important because Brownian motion, after transformation to the Ornstein-Uhlenbeck process, belongs to it. Fortunately it (along with the case $\alpha=2$ ) is the easiest to handle and we can compute $H_{\alpha}(g)$ exactly when $g$ is linear. The special fact here is that $X_{\alpha}(\tau)$ is itself Brownian motion with negative drift rate one-half. Thus we are able to obtain

LemMa 11. Let $g(\tau)=c \tau, c \geqslant 0$. Then $H_{1}(g)=\frac{1}{2}$. 
Proof. If $W(\tau)$ is a mean zero Wiener process, it is well known [11] that

$$
\begin{aligned}
P(W(\tau) & \geqslant a+b \tau, \text { some } \tau \leqslant T) \\
& =1-\Phi\left((a+b T) / T^{1 / 2}\right)+\exp (-2 a b) \Phi\left((-a+b T) / T^{1 / 2}\right) .
\end{aligned}
$$

Letting $c^{\prime}=c+\frac{1}{2}$ and remembering $X_{1}(\tau)=W(\tau)-\tau / 2$ we have

$$
\begin{aligned}
\int_{0}^{\infty} P\left(X_{1}(\tau) \geqslant\right. & s+c \tau, \text { some } \tau \leqslant T) e^{s} d s \\
= & \int_{0}^{\infty}\left[1-\Phi\left(\left(s+c^{\prime} T\right) / T^{1 / 2}\right)\right] e^{s} d s \\
& +\int_{0}^{\infty} e^{-s\left(2 c^{\prime}-1\right)} \Phi\left(\left(c^{\prime} T-s\right) / T^{1 / 2}\right) d s .
\end{aligned}
$$

An integration by parts shows that the first term in (5.2) equals

$$
\begin{aligned}
\Phi^{*}\left(c^{\prime} T^{1 / 2}\right) & +\int_{0}^{\infty} \phi\left(\left(s+c^{\prime} T\right) / T^{1 / 2}\right) e^{s} d s / T^{1 / 2} \\
& =\Phi^{*}\left(c^{\prime} T^{1 / 2}\right)+T^{-1 / 2} \int_{0}^{\infty} \phi\left(\left\{s+\left(c^{\prime}+1\right) T\right\} / T^{1 / 2}\right) e^{\left(c^{\prime}+1 / 2\right) T} d s \\
& =\Phi^{*}\left(c^{\prime} T^{1 / 2}\right)+e^{\left(c^{\prime}+1 / 2\right) T} \Phi^{*}\left(\left(c^{\prime}+1\right) T^{1 / 2}\right) \rightarrow 0 \quad \text { as } T \rightarrow \infty
\end{aligned}
$$

When $c^{\prime}=\frac{1}{2}$, the second term in (5.2) equals

$$
\int_{0}^{\infty} \int_{-\infty}^{(T / 2-s) T^{-1 / 2}} \phi(u) d u d s=\int_{-\infty}^{T^{1 / 2} / 2}\left(\frac{1}{2} T-T^{1 / 2} u\right) \phi(u) d u \sim \frac{1}{2} T
$$

so that $H_{1}(0)=\frac{1}{2}$. When $c^{\prime}>\frac{1}{2}$, a parts integration shows that the second term in (5.2) equals

$$
\begin{aligned}
\left(2 c^{\prime}-\right. & 1)^{-1}\left[\Phi\left(c^{\prime} T^{1 / 2}\right)-\int_{0}^{\infty} \exp \left\{-s\left(2 c^{\prime}-1\right)\right\} \phi\left(\frac{s-c^{\prime} T}{T^{1 / 2}}\right) d s / T^{1 / 2}\right] \\
& =\left(2 c^{\prime}-1\right)^{-1}\left[\Phi\left(c^{\prime} T^{1 / 2}\right)-\int_{0}^{\infty} \phi\left(\frac{s+\left(c^{\prime}-1\right) T}{T^{1 / 2}}\right) d s \exp \left\{-T^{1 / 2}\left(c^{\prime}-\frac{1}{2}\right)\right\}\right] \\
& =\left(2 c^{\prime}-1\right)^{-1}\left[\Phi\left(c^{\prime} T^{1 / 2}\right)-\exp \left\{-T^{1 / 2}\left(c^{\prime}-\frac{1}{2}\right)\right\} \Phi^{*}\left(\left(c^{\prime}-1\right) T^{1 / 2}\right)\right] \\
& \rightarrow\left(2 c^{\prime}-1\right)^{-1}=(2 c)^{-1} \text { as } T \rightarrow \infty .
\end{aligned}
$$

Also $\int_{0}^{\infty} \exp (-c \tau) d \tau=c^{-1}$ so that from (3.1) and (5.3) $H_{1}(c \tau)=(2 c)^{-1} / c^{-1}=\frac{1}{2}$.

Let us consider the problem of estimating

$$
P(X(t) \geqslant f(t), \text { some } t \in T)
$$

when $X(t)$ is the Ornstein-Uhlenbeck process, i.e. $\rho(t)=E X(0) X(t)=e^{-|t|}$. Thus $\sigma^{2}(t)=2(1-\exp (-|t|)) \sim 2|t|$ so that $\Delta(x) \sim \frac{1}{2} x^{-2}$. Then

$$
\begin{aligned}
g_{n}(t, \tau) & =f(t)[f(t+\tau \Delta)-f(t)] \\
& \sim f(t)\left[f\left(t+\frac{1}{2} \tau(f(t))^{-2}\right)-f(t)\right] \sim \tau f^{\prime}(t) / f(t)
\end{aligned}
$$

and it is reasonable to embed $f(t)$ in a multiplicative family so that $g(t, \tau)=$ $\tau f^{\prime}(t) / f(t)$. Now, from Theorem 1 and Lemma 11, an approximation for (5.4) is $\int_{T} f(t) \phi(f(t)) d t+\lambda_{a}^{-}+\lambda_{b}^{+}$. Notice that, as $H_{1}(g)$ is constant, $g$ is only used to 
decide if the boundary terms are nonnegligible. This result can be translated back to Brownian motion to yield the following useful formula which bears a resemblance to a result of Wichura [17]:

Corollary. Let $W(t)$ be Brownian motion. Assume $\left\{f_{n}\right\}$ are continuously differentiable on $T=[a, b]$ and that $f_{n}^{\prime} / f_{n}$ converges uniformly on compact subsets of $T \cap(0, \infty)$ as $n \rightarrow \infty$ and that $\hat{f}_{n}(t)=e^{-t} f_{n}\left(e^{2 t}\right)$ satisfies $(\mathrm{C} 2)$ and (C3). Then as $n \rightarrow \infty$

$$
\begin{aligned}
P\left(W(t) \geqslant f_{n}(t)\right. & \text { some } t \in T) \\
& \sim \int_{T}(2 t)^{-1}\left(f_{n}(t) / t^{1 / 2}\right) \phi\left(f_{n}(t) / t^{1 / 2}\right) d t+\lambda_{a}^{-}+\lambda_{b}^{+}
\end{aligned}
$$

where

$$
\begin{aligned}
& \lambda_{a}^{-}=\Phi^{*}\left(f_{n}(a) / a^{1 / 2}\right) I_{\left\{\left.\left(f_{n}(t) / t^{1 / 2}\right)^{\prime}\right|_{t-a}>0, a>0\right\}}, \\
& \lambda_{b}^{+}=\Phi^{*}\left(f_{n}(b) / b^{1 / 2}\right) I_{\left\{\left.\left(f_{n}(t) / t^{1 / 2}\right)^{\prime}\right|_{t-b}<0, b<\infty\right\}}
\end{aligned}
$$

REMARK. When $f_{n}=n f(t)$ the conditions of the corollary are satisfied if $f$ is strictly positive, continuously differentiable and

$$
\int_{T}\left(f(t) / t^{3 / 2}\right) \phi\left(f(t) / t^{1 / 2}\right) d t<\infty
$$

When $T=[0, T]$ and $f(t)=a+b t, a>0, b \geqslant 0$, differentiation of (5.1) yields the exact first passage density $p(t)=a t^{-3 / 2} \phi\left((a+b t) / t^{1 / 2}\right)$ which differs from the integrand of $(5.5)$ in that the factor $\frac{1}{2}(a+b t)$ is replaced by $a$. However $\phi\left((a+b t) / t^{1 / 2}\right)$ has a maximum at $a / b$ and the two functions agree there. If $f(t)$ is embedded in a multiplicative family $n f(t)$, then as $n \uparrow \infty$ the probability density of a crossing becomes concentrated entirely in a neighborhood of $a / b$ so the formulas are asymptotically equivalent when $T \geqslant a / b$. If $T<a / b$ the boundary term $\lambda_{T}^{+}$ must be considered. However

$$
\lambda_{T}^{+}=\Phi^{*}\left(f(T) / T^{1 / 2}\right)=\int_{0}^{T}(2 t)^{-1}\left\{(a-b t) / t^{1 / 2}\right\} \phi\left((a+b t) / t^{1 / 2}\right) d t
$$

so that, for any $0<T<a / b, \int_{0}^{T} p(t) d t=\int_{0}^{T} \lambda(t) d t+\lambda_{T}^{+}$and (5.5) is exact.

REMARK. The fundamental character of (5.5) is reinforced by the fact that the integral appearing in this expression is exactly the integral test criterion for upper and lower class functions [7, p. 33]: the finiteness of (5.5) is equivalent to there being positive probability of not crossing $f(t)$ in a neighborhood of the origin.

6. The case $\alpha=2$ and differentiable processes. When $X(t)$ has a q.m. derivative and $f^{\prime}(t)$ is continuous, the expected number of crossings of $f$ is finite. Crossing results in this case have been worked out in some detail (cf. Cramer and Leadbetter [4]). If $N(f, t)$ denotes the number of upcrossings of $f(t)$ by the process $X(t)$ in the interval $[0, T]$ then we have the following formula for the mean number of such crossings $[4$, p. 285]

$$
E N(f, T)=\int_{0}^{T} E\left(X^{\prime}(t)-t^{\prime}(t)\right)^{+} \phi(f(t)) d t .
$$


This formula bears a great resemblance to (3.5) and has been used by Kozačenko and Rudomanov [8] to obtain an equivalent form of Theorem 1 in this special case. If we normalize both time and space so that $E X^{2}(t)=1, \sigma(t) \sim t$ then $\psi(f(t)) / \Delta(f(t)) \sim \phi(f(t))$ and we might expect to equate $E\left(X^{\prime}(t)-f^{\prime}(t)\right)^{+}$with $H_{2}\left(f^{\prime}(t) \tau\right)$. When $f$ is nondecreasing this is indeed the case and Theorem 1 can be developed from (6.1) and the second moment formula [4, p. 204]

$$
\begin{aligned}
E(N(f, A) N(f, B)) & \\
=\int_{A} \int_{B} E\left(\left(X^{\prime}(t)-f^{\prime}(t)\right)^{+}\left(X^{\prime}(s)-f^{\prime}(s)\right)^{+}\right. & \mid X(t)=f(t), X(s)=f(s)) \\
& \times \phi(f(s), f(t), \rho(t-s)) d s d t
\end{aligned}
$$

where $A$ and $B$ are disjoint time intervals and $\phi(x, y, \rho)$ is the standard bivariate normal density. However a more straightforward method of evaluating $\mathrm{H}_{2}(g)$ uses the fact that $X_{2}(t)$ is the trivial process $\left(X-\frac{1}{2} t\right) t$, where $X$ is a standard normal variate.

Direct calculation from (3.1) establishes the following

LEMMA 12. $H_{2}(c \tau)=E(X-|c|)^{+}=\phi(c)-|c| \Phi^{*}(|c|)$ where $X$ is standard normal.

When $f$ is not increasing the integral in (3.5) does not equal the expected number of upcrossings or downcrossings but a mixture of the two: upcrossings when $f$ is increasing (or flat) and downcrossings when $f$ is decreasing. As a consequence, $\int_{T_{n}} \lambda_{n}(t) d t$ is never greater than the expected number of upcrossings or downcrossings. If one adds terms to account for being above the boundary at endpoints and local minima, one might expect to develop more exact approximations in terms of higher moments for boundary crossings along the lines of Cramér, Leadbetter and Serfling [5].

7. Applications. The need for estimating boundary crossing probabilities is obvious, especially in sequential analysis where the embedding of sample sums in a Wiener process is well known. For moderate sample sizes however, the passage from discrete to continuous time results in overly conservative significance levels (Siegmund [15]). Correction factors for discrete time ( $a>0$ in our notation) and a more detailed study of the applications of the above results to sample sums and Brownian motion will appear elsewhere. However, two uses of our results are of enough interest to be briefly developed here. Implicit in Theorem 1 and its extensions is the fact that $\lambda_{n}(t)+\left.(d / d T) \lambda_{T}^{+}\right|_{T=t^{-}}$is an approximation for the density of the (possibly defective) first passage time, valid when the total probability of a crossing is small. Restricting attention to Brownian motion, the density has the approximate form

$$
\left\{(2 t)^{-1} \hat{f}(t)+\left(\hat{f}^{\prime}(t)\right)^{-}\right\} \phi(\hat{f}(t))
$$

where $x^{-}=\max (0,-x)$ and $\hat{f}(t)=f(t) / t^{1 / 2}$. Also when the probability of crossing $f(t)$ is small, the location of the first crossing of $f$, conditional on the fact that a crossing occurs, is proportional to (7.1). From (7.1) we see that the case of a 
one-sided boundary of the form $f(t)=\alpha t^{1 / 2}$, studied by Armitage, McPherson [1], [9] and others, has first passage density approximately proportional to $t^{-1}$. As this is not integrable near zero, we cannot consider intervals containing the origin, in agreement with the law of the iterated logarithm. Thus under the null hypothesis of zero drift, sequential tests with a boundary of this shape are most likely to exceed it very near the origin and most of the allowable type I error occurs over a very small portion of the total time of the test. Equation (7.1) permits control of this phenomenon. For example if one wanted the first passage density to be a constant $C$, the appropriate boundary is approximately

$$
f(t)=t^{1 / 2} \chi^{-1}(2 C t)=(2 t \log 1 / t)^{1 / 2}(1+o(1))
$$

where $\chi(x)=x \phi(x)$ and $C t \ll 1$. These results are also applicable to two-sided tests, as the probability of crossing both the upper and lower boundaries is of second order.

Another application of the results is in assessing the significance of sequential trials that have reached their maximum size and not crossed a high stopping boundary. In medical trials of fixed maximum size, for ethical reasons it is necessary to have stopping rules which terminate the trial early if major treatment differences arise. Such a decision would be based on crossing a pre-established boundary and the true significance level $\alpha$ should be quite small (say $\alpha=.01$ or .02 ), as one should be very certain about a result before stopping an experiment in progress. However, if one reaches the maximum sample size without stopping one would still like to report results significant at a somewhat lesser level $\alpha^{*}$ (say $\alpha^{*}=.05$ ). Our result can be used to account for the probability of stopping early in computing $\alpha^{*}$. For simplicity again embed the random walk in Brownian motion with the maximum sample size corresponding to $t=1$. If $f(t)$ is our stopping boundary we wish to compute

$$
\begin{aligned}
\alpha^{*}(z) & =P(W(t) \geqslant f(t) \text { some } t \leqslant 1 \text { or } W(1) \geqslant z) \\
& =P(W(1) \geqslant z)+P(W(t) \geqslant f(t) \text { some } t \leqslant 1, W(1)<z) .
\end{aligned}
$$

Letting $p(t)$ denote the first passage density and using the strong Markov property (7.2) equals

$$
\begin{gathered}
\Phi^{*}(z)+\int_{0}^{1} P(W(1)<z \mid W(t)=f(t)) p(t) d t \\
=\Phi^{*}(z)+\int_{0}^{1} \Phi\left(\frac{z-f(t)}{(1-t)^{1 / 2}}\right) p(t) d t
\end{gathered}
$$

and we may approximate this by replacing $p(t)$ with (7.1).

ACKNOWLEDGEMENT. I am grateful to the referee for comments leading to improvements of Lemma 3 and example (iv).

\section{REFERENCES}

1. P. Armitage, Sequential medical trials, 2nd ed., Blackwell, Oxford, 1975.

2. S. M. Berman, Excursions of stationary Gaussian processes above high moving boundaries, Ann. Probability 1 (1973), 365-387.

3. __ Sojourns and extremes of Gaussian processes, Ann. Probability 2 (1974), 999-1026. 
4. H. Cramér and M. R. Leadbetter, Stationary and related stochastic processes, Wiley, New York, 1967.

5. H. Cramér, M. R. Leadbetter and R. J. Serfling, On distribution function moment relationships in a stationary point process, Z. Wahrscheinlichkeitstheorie und Verw. Gebiete 18 (1971), 1-8.

6. X. Fernique, Continuité des processus Gaussiens, C. R. Acad. Sci. Paris Sér. A-B 258 (1964), 6058-6060.

7. K. Itô and H. P. McKean, Diffusion processes and their sample paths, Springer, Berlin, 1974.

8. Ju. V. Kozačenko and V. I. Rudomanov, On the probability that a stationary random process exceeds a given function, Theory Prob. Math. Statist. 12 (1976), 65-78.

9. C. K. McPherson and P. Armitage, Repeated significance tests on accumulating data when the null hypothesis is not true, J. Roy. Statist. Soc. Ser. A 134 (1971), 15-26.

10. C. Qualls and H. Watanabe, Asymptotic properties of Gaussian processes, Ann. Inst. Math. Statist. 43 (1972), 580-596.

11. H. Robbins and D. Siegmund, Statistical tests of power one and the integral representation of solutions of certain partial differential equations, Bull. Inst. Math. Acad. Sinica 1 (1973), 93-120.

12. J. Pickands, Upcrossing probabilities for stationary Gaussian processes, Trans. Amer. Math. Soc. 145 (1969), 51-73.

13. V. I. Piterbarg and V. P. Prisjažnjuk, Asymptotic behavior of the probability of a large excursion for a nonstationary Gaussian process, Teor. Verojatnost. i Mat. Statist. 18 (1978), 121-134.

14. E. Seneta, Regularly varying functions, Lecture Notes in Math., vol. 508, Springer, Berlin, 1976.

15. D. Siegmund, Repeated significance tests for a normal mean, Biometrika 64 (1977), 177-189.

16. D. Slepian, The one-sided barrier problem for Gaussian noise, Bell System Tech. J. 41 (1962), 463-501.

17. M. J. Wichura, Boundary crossing probabilities associated with Motoo's law of the iterated logarithm, Ann. Probability 1 (1973), 437-456.

Cancer Epidemiology and Clinical Trials Unit, University of Oxford, Oxford OXI 3QG, ENGLAND (Current address)

Department of Mathematical Statistics, Columbia University, New York, New York 10027 